\title{
Oclusivas complejas en el quechua de Domingo de Santo Tomás
}

\section{Complex Stops in the Quechua of Domingo de Santo Tomás}

\section{Simeon Floyd}

Universidad San Francisco de Quito, Quito, Ecuador

Contacto: sfloyd1@usfq.edu.ec

https://orcid.org/0000-0002-9739-2914

\section{RESUMEN}

Tradicionalmente, el quechua descrito por Domingo de Santo Tomás en 1560 se ha contrastado con el quechua cuzqueño por la ausencia de una representación ortográfica para oclusivas complejas y por la presencia de sonorización posnasal de oclusivas escrita como $\langle\mathrm{b}\rangle,\langle\mathrm{d}\rangle \mathrm{y}\langle\mathrm{g}\rangle$. Previamente no ha sido posible explicar por qué el autor escribe estas letras con aparente irregularidad, usándolas en algunos casos, pero usando $\langle\mathrm{p}\rangle$, $\langle\mathrm{t}\rangle \mathrm{y}\langle\mathrm{c}\rangle$ en otros. Este estudio demuestra que dicha alternancia no es irregular, sino que el uso de $\langle b d g\rangle$ versus $<$ ptc $>$ corresponde regularmente a la distinción de oclusivas simples versus complejas en quechuas modernos que cuentan con estos sonidos, como el quechua cuzqueño-boliviano. Este resultado de alta significancia estadística sugiere que el quechua descrito por Santo Tomás sí tenía contrastes entre consonantes complejas y simples, y que la sonorización alofónica de oclusivas después de /n/ se limitaba a palabras con oclusivas simples. Tomando en cuenta que escritores españoles coloniales tardaban unas generaciones en lograr escribir sonidos de lenguas americanas que no existían en el español, se concluye que las aspiradas y ejectivas estaban presentes en el quechua de Santo Tomás, pero que no se escribían todavía durante el siglo XVI, solamente desde inicios del siglo XVII. Esta evidencia ortográfica colonial que señala oclusivas complejas en un quechua hablado lejos de Cuzco indica que estos sonidos podrían ser retenciones antiguas en las lenguas quechuas.

Palabras clave: Lenguas quechuas; Domingo de Santo Tomás; Oclusivas complejas; Ortografía

\section{ABSTRACT}

Traditionally the Quechua described by Domingo de Santo Tomás in 1560 has been contrasted with Cuzco Quechua due to the absence of an orthographic representation of complex stops and to the presence of postnasal voicing of stops written as $\langle b\rangle,\langle d\rangle$, and $\langle g\rangle$. Previously it has not been possible to explain why the author apparently wrote these letters irregularly, sometimes using them but sometimes using $\langle\mathrm{p}\rangle,\langle\mathrm{t}\rangle$, and $\langle\mathrm{c}\rangle$. This study shows that this alternation is not irregular, but that the use of $\langle$ bdg $>$ versus $<$ ptc $\rangle$ corresponds regularly to the distinction of simple versus complex stops in modern Quechuan languages that have these sounds, like Cuzco-Bolivian Quechua. This highly statistically significant result suggests that the Quechua described by Santo Tomás did have a contrast between complex and simple consonants, and that allophonic voicing of stops after /n/ was limited to words with simple stops. Taking into account that Colonial Spanish writers took a few generations to be able to write sounds of indigenous American languages that did not exist in Spanish, we can conclude aspirates and ejectives were present in Santo Tomas's Quechua, but that they were not written yet during the $16^{\text {th }}$ Century, only from the beginning of the $17^{\text {th }}$. This Colonial orthographic evidence pointing to complex stops in a Quechua spoken far from Cuzco indicates that these sounds may be older retentions in the Quechuan languages.

Keywords: Quechuan Languages; Domingo de Santo Tomás; Complex stops; Orthography 


\section{Introducción y antecedentes}

Con base en las prácticas ortográficas observadas en la escritura quechua de las obras de Domingo de Santo Tomás Grammatica o arte de la lengua general de los Indios de los Reynos del Peru y Lexicón o Vocabulario de la lengua general del Pero (ambos del mismo año; 1560a, 1560b), tradicionalmente se ha considerado que estas se distinguen del quechua cuzqueño en dos elementos principales: la aparente ausencia de oclusivas complejas o laringalizadas, debido a la falta de una práctica ortográfica consistente para representarlas, y la presencia de sonorización posnasal, determinada basándose en el uso de los grafemas $<\mathrm{b}>,<\mathrm{d}>$ y $<\mathrm{g}>$ después de consonantes nasales $<\mathrm{n}>(<\mathrm{m}>$ antes de bilabiales) (Parker, 1963, pp. 249-250; Torero, 1964, pp. 448, 451, 463-465, 474-475). Este estudio presenta un nuevo análisis de la ortografía de Domingo de Santo Tomás (desde ahora, "DST") que sugiere que el quechua que transcribía en realidad tenía oclusivas complejas contrastivas, y que estas se reflejan sistemáticamente en las alternaciones de la sonorización posnasal en esta lengua. Una comparación del material léxico con lenguas quechuas modernas demostró una tendencia altamente significativa por parte de DST a usar los grafemas <bdg $>$ posnasales solamente en palabras cuyos cognados en quechua cuzqueño no tienen oclusivas aspiradas o eyectivas, mientras que palabras con oclusivas complejas son escritas con $\langle$ ptc $>$.

A fines del siglo XVI, la práctica de escribir $<$ bdg $>$ en quechua desaparece casi por completo después de la publicación de los materiales extensivos del Tercer Concilio Limense (Anónimo, 1584; 1586) que no hacían uso de estas letras, aunque sí se nota que todavía se escuchaban pronunciaciones sonorizadas en esta época: "los que hablan corruptamente esta lengua mudan la ca, qui, en ga gui, como Inga, ringui, por ynca, rinqui” (Anónimo, 1584, p. 75). En estos documentos vemos referencias a zonas donde no hablan "con la perfeccion que en el Cuzco", pero se explica que "de dos extremos se ha procurado huyr" en los materiales del Tercer Concilio; se evita ser demasiado específico a Cuzco o demasiado generalizado al resto del territorio para que sea conforme con "lo que se vsa desde Quito hasta los Charcas" (Anónimo, 1584, p. 75; véase también Ezcurra Rivero y Bendezú-Araujo, 2017).
En estos documentos también vemos la primera mención de las oclusivas complejas consideradas características del quechua cuzqueñoboliviano moderno, "pronunciandolas los Indios mas asperamente o mas blandamente conforme a lo que quieren significar," aunque se decide no representarlas ortográficamente porque "ni concuerdan con las significaciones ni convienen los interpretes, entre si" (Anónimo, 1583, p. 75). No será por algunas décadas más hasta que González Holguín (desde ahora, "GH") aplicaría la estrategia de duplicación de letras para escribir las oclusivas complejas (1607; 1608), aunque de forma inconsistente, como veremos más abajo. Es solamente alrededor de 1648 que la ortografía de GH sería adoptada generalmente, estableciendo una norma hasta las primeras décadas del siglo XX (Itier, 1995, p. 123).

La ausencia de una representación ortográfica de oclusivas complejas en los textos de DST junto con la presencia de <bdg $>$ en estos y otros documentos tempranos ha sido interpretada como un indicador de dos sistemas fonológicos paralelos muy distintos, uno de un quechua "costeño" o "general" representado por los documentos de DST (1560) y algunos otros pocos ejemplos de textos cortos tempranos como los de Betanzos (Betanzos, 1880 [1551]), Cieza de León (1553)y Quiroga (1992 [1569]; véase también CerrónPalomino, 2017), y otro del quechua cuzqueño, representado en la mayoría de los documentos que siguieron (véase Ezcurra Rivero y Bendezú-Araujo, 2017 para la cronología general). Sin embargo, vale acordarnos que estos dos sistemas nunca se registraron simultáneamente en el mismo período histórico, sino secuencialmente; el sistema con $<$ bdg $>$ en alternancia con $\langle$ ptc $\rangle$ desaparece hacia finales del siglo XVI, y poco a poco surge un sistema con $<p p>$ $<\mathrm{tt}>,<\mathrm{cc}>/<_{\mathrm{qq}}>$ y $<\mathrm{chh}>$ durante el siglo XVII.

Numerosos aspectos de la obra quechua de DST han sido investigados en la literatura (particularmente en Cerrón-Palomino, 1990). Sin embargo, queda sin explicar un curioso detalle sobre el uso de $<b d g>$ posnasal en el quechua de DST: en las lenguas quechuas modernas con tendencia a la sonorización posnasal, las variantes de los grupos ecuatoriano-colombiano y Chachapoyas-Lamas, esta normalmente afecta a toda o casi toda oclusiva posnasal (además de las africadas, muchas veces; cfr. Carpenter, 1982; Stark y Muysken, 1977; Taylor, 
1979 y 2000), mientras que DST transcribía <bdg> de forma variable, en algunas raíces sí (43\%), pero en la mayoría de casos no (57\%). De más de 100 palabras con $/ \mathrm{n} /$ seguida por una oclusiva, existen en total solamente cinco casos de variación ortográfica de $<$ ptc $>$ y $<$ bdg $>$ después de /n/: <anco $>$, <ango $>$ 'águila'; <runto $>$, < rundo $>$ 'granizo'; < chollonco $>$, $<$ chollongo > 'hielo'; <comba>, <compa> 'bordar'. Aunque DST menciona que algunos hablantes varían "b por p" en el prólogo de su Vocabulario (1560b, p. 6), no parece registrar este tipo de variación principalmente. Más bien, en todos los otros casos, cada raíz se escribe de una sola forma consistente en toda la obra de DST.

¿por qué motivo hubiera decidido DST reemplazar tantas palabras locales con una pronunciación de otro lado, llegando a afectar más de la mitad del total de palabras con oclusivas posnasales?

Antes de descartar la evidencia relevante como una irregularidad o una introducción foránea, es posible primero verificar si las alternancias se motivan por factores lingüísticos. En las siguientes secciones demostraremos que la variación observada en DST no es irregular, sino que corresponde regularmente a la distinción entre palabras con y sin oclusivas complejas en los quechuas modernos que las tienen.

\section{Métodos y materiales}

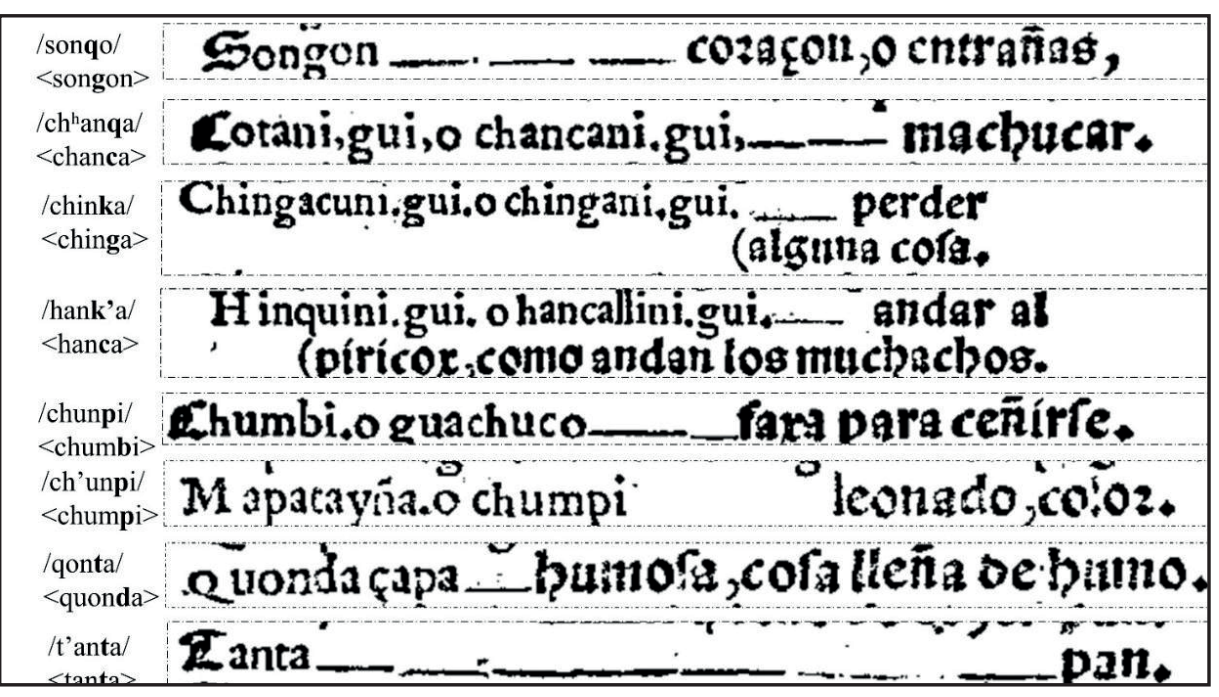

Figura 1.

Alternancia ortográfica de DST entre $<$ ptc $>$ y $<$ bdg $>$ después de /n/, comparado con la pronunciación cuzqueña moderna.

¿Cómo explicar esta aparente sonorización que solamente afecta a una minoría de palabras, y no es generalizada? Se ha sugerido que podría ser resultado de préstamos de algún otro quechua (Cerrón-Palomino, 1990, p. 349; Parker, 1969, p. 152) o que podría representar un cambio fonológico ocurriendo en ese entonces (Cerrón-Palomino, 1990, p. 349), pero ninguna de estas propuestas logra predecir cuáles palabras se sonorizarían y cuáles no. En el caso de la propuesta de cambio fonológico, ¿por qué esto afectaría consistentemente estas raíces y las otras no? Los cambios regulares de sonidos afectarían el sistema fonológico en general, no solamente algunas palabras específicas sin una motivación fonológica. $\mathrm{O}$, en la propuesta de préstamos, si la mayoría de estas palabras ya hubiesen existido en todos los quechuas,
En primer lugar, se recopiló una lista completa de todas las palabras con ocurrencias posnasales de grafemas $<b d g>$ versus $<$ ptc $>$ $(\mathrm{o}<\mathrm{qu}>$ ) en el Vocabulario y la Grammatica de DST, contando cada raíz una sola vez. Después, se contrastaron estos datos con palabras cognadas en lenguas quechuas actuales que cuentan con fuentes con transcripción fonética moderna. La hipótesis del estudio fue que la alternancia sorda-sonora en el quechua de DST tenía una motivación lingüística que podría reflejarse en un quechua moderno que se podría detectar usando el método comparativo.

Primero, para determinar si se vería alguna alternancia similar a la observada en DST en lenguas quechuas modernas con sonorización posnasal, se comparó la lista de palabras con el quichua serrano ecuatoriano (dialecto de Quito como base) y el quechua de Chachapoyas-Lamas. Sin embargo, estas lenguas sonorizan en todos o casi todos los $\operatorname{casos}^{1}$. Con el propósito de comparar también con una lengua quechua sin sonorización posnasal notable, la tercera lengua comparada con el quechua de DST fue el quechua cuzqueño actual, con base en fuentes modernas $^{2}$, con información histórica suplementaria de fuentes coloniales como el Vocabulario del siglo XVII de GH (1608). El Vocabulario de GH tiene una mayor cercanía temporal a los documentos de DST, pero desafortunadamente no se lo puede usar de 
manera exclusiva porque el autor escribe letras dobles de una forma consistente solamente $42 \%$ de las veces, basándose en una muestra léxica (400 palabras; n $=257$ con oclusivas; véase abajo para detalles de la lista), y no hay suficiente coincidencia con el léxico relevante de DST para una comparación cuantitativa directa entre estas fuentes.

La lista completa de palabras de DST contiene 108 raíces léxicas únicas con oclusivas posnasales, $80 \%$ de las cuales tienen cognados en las otras lenguas quechuas consideradas y satisficieron todos los requisitos para ser comparadas con ellas. El análisis cuantitativo se hizo con base en esta lista $(\mathrm{n}=$ 86). Se usó estadística básica con una prueba clásica chi cuadrado (Pearson, 1900) con una corrección de continuidad (Yates, 1934), junto con una prueba exacta de Fisher (1922) para evaluar si existía una correspondencia sistemática entre la alternancia entre $<$ ptc $>$ y $<$ bdg $>$ en DST y algún contraste de sonidos en las otras lenguas quechuas.

Estas cifras no incluyen los 4 sufijos gramaticales que tienen consonantes posnasales $\mathrm{u}$ otros sufijos que ocurren con raíces que terminan en nasal. La tendencia ahí es a escribir $\langle\mathrm{g}>$ después de nasales dentro de morfemas (<-ngui $>,<$-nga $>$ ) pero no en fronteras morfológicas (<-ca $>,<$-cama $>$ ), y a nunca usar $<$ b $>$ o $<$ d $>$ (-manta, -ta, -ntin, -pa, -pac, -pas, -pi). Estos casos están registrados en el apéndice, pero el análisis presentado aquí se basa en las raíces léxicas.

Además de estos casos, también se recopiló algunos ejemplos de $<\mathrm{b}>\mathrm{y}<\mathrm{d}>$ en otros contextos dondenoparecesernecesariamenteunarepresentación de una oclusiva sonora, en unos casos representando un sonido epentético (< guambra > 'joven', < hambra $>$ 'atinar', <rambra> 'álamo' o 'aliso') y en otro caso se ubica al final de una palabra que no parece ser de origen quechua (<catquid $>$ 'frente'). También vemos una serie de palabras escritas con $\langle$ b $>$ y $<$ d $>$ en posición final de la sílaba: <habra $>$, <guabci $>$, <çabra>, <quessibra>/<quexibra>, <cadqui>, $<$ tadqui $>$, < $<$ vidco $>$. Distintas de las posnasales, estos usos de $<$ bdg $>$ no parecen indicar la sonorización sino el desgaste (o lenición) de la consonante (CerrónPalomino, 1990, pp. 351-353). Aunque estos ejemplos no fueron incluidos en el análisis estadístico, están presentados aquí en un apéndice que contiene la base de datos completa de todos los usos de los caracteres $<$ bdg $>$ en la obra de DST. La siguiente sección presenta los resultados cuantitativos.

\section{Resultados}

Como se mencionó anteriormente, no se encontraron correspondencias relevantes entre el quechua de DST y las dos otras lenguas quechuas consideradas en adición al cuzqueño; en las tablas de abajo se incluye un dialecto del quichua serrano ecuatoriano como un ejemplo. En cambio, cuando se comparó con el quechua cuzqueño se identificaron correspondencias con la alternancia entre $<$ ptc $>$ y $<$ bdg $>$ en las obras de DST: resulta que las palabras con $<$ bdg $>$ posnasal tienen cognados con oclusivas sencillas en el quechua cuzqueño, mientras que las que tienen <ptc> posnasal tienen cognados con aspiradas o eyectivas en el cuzqueño. Aunque esta relación es una tendencia generalizada, no una regla universal, es una tendencia muy fuerte $(84 \%$ correspondencia /p/, /t/, /k/ y /q/ en conjunto, y $90 \% / \mathrm{k} / \mathrm{y} / \mathrm{q} /$ solamente).

Empezando con las palabras escritas con $<\mathrm{g}>$ después de /n/ en DST, encontramos que la mayoría de sus cognados en quechua cuzqueño tenían oclusivas simples $(19 / 21)^{3}$. El quichua ecuatoriano también sonoriza oclusivas en todos los casos donde se pudo encontrar cognados, aunque hay muchas menos correspondencias léxicas en general que cuando comparamos el quechua de DST y el cuzqueño.

Por otro lado, comparando con las palabras que DST escribe con /c/, en la tabla 2 se ve que la gran mayoría corresponde a palabras que llevan oclusivas complejas (20/23). En la mayoría de estos casos, las oclusivas posnasales se sonorizarían en lenguas como el quichua ecuatoriano y el quechua de Chachapoyas, pero en el quechua de DST esto no ocurre. 


\begin{tabular}{|c|c|c|c|}
\hline Definición DST & Palabra DST & $\begin{array}{c}\text { Quechua Cuzco mod. } \\
\text { (e histórico GH) }\end{array}$ & Quichua ec \\
\hline abejon, falsa abeja & acatanga & akatanqa (acatancca) & NA \\
\hline aguila, aue conoscida & anga, anca, ancca & anka (anca, ancca) & anga \\
\hline azul color & angax & anqas (ancas) & NA \\
\hline assar & canga- & kanka- (canca-) & NA \\
\hline desaparecer & chinga- & chinka- (chinca-, chincca-) & chinga \\
\hline diez & chunga & chunka (chunca, chuncca) & chunga \\
\hline naríz & cinga & sinqa (cenca, cencca) & singa \\
\hline elada, o yelo & chollungoy, cholloncoy & chullunku, chhullunku, chullunckuy & NA \\
\hline rodilla & congor & qunqur (concor, cconcor, cconccor) & kunguri \\
\hline cuello & conga- & kunka- (cunca-, cuncca-) & kunga \\
\hline mil & guaranga & waranqa (huaranca, huarancca) & waranga \\
\hline Inca & inga, ynga & inka (inca) & inga, inka \\
\hline tigre & otorongo & uturunku (vturuncu) & NA \\
\hline flauta & pingollo & pinkuyllu (pincullu) & pinguzhu \\
\hline puerta & pongo & punku (puncu) & pungu \\
\hline hinchar & pungui- & punki- (punqui-) & pungi \\
\hline coraçón o entrañas & songo & sunqu (sonco, soncco) & shungu \\
\hline empujar & tanga-, tanca- & $\begin{array}{l}\text { tanqa- (ttancca-, ttanca-, } \operatorname{tancca-}, \\
\text { tanca-) }\end{array}$ & tanga \\
\hline garganta & tongorí & tunqur (toncor, ttoncor, tonccori) & tunguri \\
\hline falso & yanga & yanqa (yanca, yancca) & yanga \\
\hline
\end{tabular}

Tabla 1. Palabras con la secuencia $<$ ng $>$ en el quechua de DST y con oclusivas simples en el quechua cuzqueño $(n=20)$.

\begin{tabular}{|c|c|c|c|}
\hline Definición DST & Palabra DST & $\begin{array}{c}\text { Quechua Cuzco mod. } \\
\text { (e histórico GH) }\end{array}$ & Quichua ec \\
\hline nieruo, o vena de animal & anco, hanco & hank'u (anccu, ancco) & angu \\
\hline atormecerse & çaçunca, cuçunca, çoçonca & susunkha (çuçuncay) & NA \\
\hline espesar & çanco- & sankhu- (çanccu-) & NA \\
\hline machucar & chanca- & chhanqa- (chamcca-, chancca-) & NA \\
\hline columpiar & guaylluncu & wayllunk'u (huaylluncu) & NA \\
\hline aduce o atambor & guancar & wank'ar (huancar) & wankar (raro) \\
\hline açucena & hamancáy & hamanq'ay (hamancay) & NA \\
\hline $\begin{array}{l}\text { coxo de pie, derrengar, } \\
\text { deslomar, andar piricox }\end{array}$ & hanca & hank'a (hancca, hanca) & hanga \\
\hline boca arriba & hancarca, ancalla & hanq'a, hant'a (hantta, hanta) & NA \\
\hline tostar & hamca- & hank'a- (hamcca-, hamca-) & NA \\
\hline en puntillas & hinqui & hink'i (hinqqui, hinqui) & NA \\
\hline tentar, atentar tocando & Ilanca-, Ilamca- & Ilankhu- (Ilamcca-, Ilamca-, Ilamka-) & NA \\
\hline acicalar & Ilonco- & Ilunk'u- (llunccu-, Iluncu-) & NA \\
\hline remolino de viento pequeño & pillonco & (ppilluncuy, pilluncuy) & NA \\
\hline verguença & pinca- & p'enqa- (ppencca-) & pinga \\
\hline abismo, agua sin ondon & poyunco & p'oyonqo (ppoyoncco) & NA \\
\hline descuydo & ranca, ranco & ranqha, rankha (rancca) & NA \\
\hline tauano & tancayllo & t'ankayllu (ttamcayllo) & NA \\
\hline papirotes dar & tinca- & t'inka- (ttinca) & NA \\
\hline apretar uno al otro & tinqui- & t'inki- (ttinqqui-, ttinqui-, -tinqqui) & NA \\
\hline
\end{tabular}

Tabla 2. Palabras con la secuencia $<$ nc $>$ en el quechua de DST y con oclusivas complejas en el quechua cuzqueño $(n=20)$. 
Las palabras con reflejos de /k/ versus /q/ fueron agrupadas por separado en un análisis preliminar, pero al no mostrar una diferencia notable con respecto a las tendencias identificadas aquí, las consideramos juntas, dejando la cuestión de la presencia de la /q/ para otro momento. Resumiendo los resultados hasta ahora, lo que parece ocurrir con los reflejos de /k/ y /q/ posnasal en la escritura de DST, según estos datos, es lo siguiente: cuando escribía $<\mathrm{g}>$, esto reflejaba una oclusiva sencilla posnasal en el quechua cuzqueño. En cambio, cuando Santo Tomás escribía $<\mathrm{c}>($ o a veces $<\mathrm{qu}>$ ), la mayoría de las palabras tiene una correspondencia cuzqueña con oclusiva compleja (39/44 casos o 90\% de correspondencia). Es decir, DST casi siempre escribía como sordas las consonantes posnasales en palabras con oclusivas complejas, y, en ausencia de laringalización, escribía las oclusivas simples como sonoras. En algunos casos como $<$ hamca $>$ se ve la /h/ protética-epentética, un proceso regular en palabras con oclusivas complejas en el quechua cuzqueño (Itier, 2016, p. 320), que también encaja con la idea de que la consonante podría haber sido una oclusiva compleja históricamente. La variación observada en algunas otras palabras $(<$ anco $>/<$ hanco $>$, $<$ hancarca $>/<$ ancalla $>$, y similar) también podría indicar un efecto de harmonía laringal de este tipo.
Vale notar que esta relación con el uso de $<c>$ no aplica solamente en las palabras con una oclusiva compleja en la consonante posnasal, sino también cuando aparece en la consonante inicial de la palabra cuzqueña: <pinca> /p'enqa/, <pollonco> /p'oyonqo/, <tinca> /t'inka/, <tinqui> /t'inki/. Esto parece relacionarse con la existencia de restricciones de larga distancia de coocurrencia en el quechua sureño, por ejemplo una que no permitiría una segunda oclusiva compleja en la palabra (CerrónPalomino, 2003, pp. 118-121; Parker y Weber, 1996). En contextos experimentales, los hablantes de quechua sureño moderno corrigen estímulos con oclusivas complejas dobles, eliminando una de las dos $\left(\right.$ Gallagher, 2013) ${ }^{5}$. Por estas razones, algunos prefieren describir la laringalización como elemento de la raíz, no el fonema individual (e. g. Parker y Weber, 1996).

Las tendencias vistas con los reflejos de las velares-uvulares parecen claras, pero, antes de interpretarlas, se debe tomar en cuenta la situación completa con todas las oclusivas. En el caso de las palabras que DST escribe con $<b>$, otra vez se ve una correspondencia fuerte a palabras que no tienen oclusivas complejas en el quechua cuzqueño $(11 / 13)^{6}$.

\begin{tabular}{|c|c|c|c|}
\hline Definición DST & Palabra DST & $\begin{array}{c}\text { Quechua Cuzco mod. } \\
\text { (e histórico GH) }\end{array}$ & Quichua ec \\
\hline ante ayer & canimba & qayninpa (canimpa, ccanimpa) & NA \\
\hline porra & chambi & chanpi (champi, chhampi) & NA \\
\hline allende el rio & chimba & chinpa (chinpa, chimba) & chimba \\
\hline arremangar & chimballi- & (chimpalli-) & NA \\
\hline vaso grande & chumba & chunpa (chumpa) & NA \\
\hline faxa & chumbi & chunpi (chumpi) & chumbi \\
\hline bordar, poner orilla & cumbi- & qonpi- (cumpi-, ccumpi-) & NA \\
\hline desembarcar, balsa & guambo-, guamboro & wanpuru (huampu-, huampo-) & wambu \\
\hline curar & hambi & hanpiy (cumpi, ccumpi) & hambi \\
\hline caço de heirro, caçuela & $\begin{array}{l}\text { tócoychimbo } \\
\text { (palabra compuesta; solo } \\
\text { comparar 'chimbo') }\end{array}$ & $\begin{array}{l}\text { (tóccoychimpo, ttocoychimpu, } \\
\text { tocoy chimpu) }\end{array}$ & NA \\
\hline desinfamar & tumba & tunpa (tumpa) & NA \\
\hline
\end{tabular}

Tabla 3. Palabras con la secuencia $<\mathrm{mb}>$ en el quechua de DST y con oclusivas simples en el quechua cuzqueño $(n=11)$. 


\begin{tabular}{|l|l|l|l|}
\hline Definición DST & Palabra DST & \multicolumn{1}{|c|}{$\begin{array}{c}\text { Quechua Cuzco mod. } \\
\text { (e histórico GH) }\end{array}$} & Quichua ec \\
\hline hongo o xeta & callampa & k'allanpa (callampa) & kazhamba \\
\hline floxo, descuydado & çampa & sanp'a (çamppa, çampa) & NA \\
\hline leonado color & chumpi & ch'unpi (chhumpi, chumpi) & NA \\
\hline sapo, rana & hampato & hanp'atu (hamppatu, hampatu) & hambatu \\
\hline color & limpi & Ilimp'i (llimppi, llimpi) & NA \\
\hline blando & llampo & llamp'u (llamppu, llampu) & zhambu \\
\hline decaido, por el frio & ompo & unphuy (vmppu, vmpu) & NA \\
\hline desmelenado & tampa & t'anpa (ttamppa, ttampa, tampa) & tamba, thamba \\
\hline hervir & timpo- & t'inpu- (ttimpu, timpu) & timbu, thimbu \\
\hline
\end{tabular}

Tabla 4. Palabras con la secuencia $<m p>$ en el quechua de DST y oclusivas complejas en el quechua cuzqueño $(n=9)$.

En cambio, en el caso del uso de $<p>$ después de $<$ m $>$, vemos que estas palabras tienden a tener oclusivas complejas (9/11). Otra vez, como con las uvulares-velares, vemos una serie de ejemplos de casos con la primera consonante compleja en vez de la última (/k'allanpa/, /ch'unpi/, etc.); es posible que una restricción de coocurrencia podría haberse aplicado en algún momento histórico para eliminar la laringalización o pasarla de una sílaba a otra. También vemos aquí que las oclusivas posnasales en estas palabras son sonoras en lenguas como el quichua ecuatoriano (a pesar de la presencia de oclusivas complejas en algunas).
El caso de <chumpi>, comparado con $<$ chumbi $>$ visto arriba (tabla 3), nos daría un par mínimo claro: <chumbi> /chunpi/ 'faja' versus <chumpi> /ch'unpi/ 'color'. Para las bilabiales, la tendencia de escribir palabras con oclusivas simples con $\langle$ b $>$ y aquellas con oclusivas complejas con $\langle p\rangle$ es casi tan grande como el patrón visto arriba con las velares-uvulares (20/23 o $87 \%$ ).

Comparado a /k/, /q/ y /p/ existen menos ejemplos con la alveo-dental /t/ después de /n/ en la obra de DST, pero aquí también vemos el mismo patrón: las palabras con $<\mathrm{d}>$ tienen oclusivas simples en el quechua cuzqueño (6/7), ilustrado en la tabla 5.

\begin{tabular}{|l|l|l|l|}
\hline Definición DST & Palabra DST & \multicolumn{1}{|c|}{$\begin{array}{c}\text { Quechua Cuzco mod. } \\
\text { (e histórico GH) }\end{array}$} & Quichua ec \\
\hline condor & condor & kuntur, kundur (cuntur) & kundur \\
\hline humear & conda-, quonda- & qonta- & NA \\
\hline sol & indi, yndi, etc. & inti (inti) & indi \\
\hline afeytarse con colorado & mandora & (mantur) & NA \\
\hline intercambiar & randi-, ra^di- & ranti- (ranti-, rantti-) & randi \\
\hline granizo & rundo, runto & runtu (runtu) & rundu \\
\hline
\end{tabular}

Tabla 5. Palabras con la secuencia $<$ nd $>$ en el quechua de DST y oclusivas simples en el quechua cuzqueño $(n=6) 7$. 


\begin{tabular}{|l|l|l|l|}
\hline Definición DST & Palabra DST & \multicolumn{1}{|c|}{$\begin{array}{c}\text { Quechua Cuzco mod. } \\
\text { (e histórico GH) }\end{array}$} & Quichua ec \\
\hline guirnalda & chanta & ch'anta (chhanta, chanta) & NA \\
\hline leña & Ilanta, Ilamta & Ilant'a (Ilamtta, yamtta, llamta) & yanta, yanda \\
\hline sombra & Ilanto & Ilanthu (Ilantu, lantu) & Ilandu \\
\hline tela & manta & mant'a (mantta, manta) & manta, manda \\
\hline mariposa & pilpinto & phillpintu, pilpinto (pilpintu) & pilpintu \\
\hline embolver & pinto- & p'intu- (ppinttu-, ppintu-, pinttu-, pintu-) & phindu, findu \\
\hline pan & tanta, tamta & t'anta (ttanta, ttantta) & tanda \\
\hline
\end{tabular}

Tabla 6. Palabras con la secuencia $<$ nt $>$ en el quechua de DST y oclusivas complejas en el quechua cuzqueño $(n=7)$.

Para este análisis se consideró principalmente oclusivas complejas seguidas por /n/, no necesariamente otros fonemas nasales, y por lo tanto dos ejemplos no fueron incluidos: sumtu con bilabial y quincti con aparente nasal velar. La falta de asimilación de las nasales en estos casos parecería indicar una oclusiva compleja históricamente, pero actualmente q'inti sí tiene una, mientras que suntu no. Podríamos preguntar si la diferencia entre /n/ y otras nasales podría explicar algo sobre la variación vista aquí, pero si fueron solamente las palabras históricamente con /m/ donde no aparece la sonorización, no veríamos los 30 ejemplos de palabras históricamente con /n/ sin sonorización en los textos de $\mathrm{DST}^{8}$; la sonorización parece reflejar otro factor distinto de la calidad de la consonante nasal anterior.

En los casos con /p/, cuando la distinción entre $/ \mathrm{n} / \mathrm{y} / \mathrm{m} /$ es neutralizada, las palabras fueron incluidas, porque no es posible distinguir diferentes nasales en esta condición. La tabla 6 demuestra que, cuando DST escribió $<\mathrm{t}>$ después de $<$ n $>$, la mayoría de casos otra vez tiene oclusivas complejas en el quechua cuzqueño (7/12). Entonces, para /t/ también vemos la misma tendencia que con los otros sonidos, con una correspondencia un poco menor (13/19 o 68,5\%). La tabla 7 presenta las distribuciones cuantitativas individuales.
Las tendencias generales de los datos se resumen en la tabla 7: en todos los casos existe una mayoría de correspondencias entre el quechua cuzqueño y el quechua de DST. Si evaluamos los datos estadísticamente, aplicando una prueba clásica Pearson de chi cuadrado con la corrección de continuidad de Yates, encontramos una asociación significante entre tipo de oclusiva, simple o compleja, y opción ortográfica, $\langle$ ptc $>$ versus $<b d g>((\chi 2,1$, $\mathrm{n}=86)=36,87, \mathrm{p}=<0,00001)$. La inspección del coeficiente phi indica que la asociación es muy fuerte $(\varphi=0,68)$. Alternativamente, si optamos por la prueba exacta de Fisher por el tamaño pequeño de la muestra, encontramos el mismo nivel de significancia estadística muy alta $(\mathrm{p}<0,00001)$.

Este resultado señala que la alternancia ortográfica de DST corresponde a la presencia y ausencia de oclusivas complejas en el quechua cuzqueño-boliviano moderno. El efecto no es total, pero nunca podríamos esperar que sea universal, porque entre los dialectos actuales del quechua sureño existe cierta variación en la distribución de las oclusivas complejas en el léxico (Carbajal Solís, 2004; Cusihuamán, 1976; Escobar Zapata, 2019; Gallagher, 2013; Gobierno Regional Cusco, 2005; Laime Ajacopa et ál., 1997; Parker y Weber, 1996; Stark, 1985). La variación observada entre el quechua de

\begin{tabular}{|l|c|c|c|}
\hline Grafemas & $\begin{array}{l}\text { [+ Oclusiva } \\
\text { compleja] }\end{array}$ & $\begin{array}{l}\text { [- Oclusiva } \\
\text { compleja] }\end{array}$ & $\begin{array}{l}\text { \% Correspondencia } \\
\text { DST - Cuzco }\end{array}$ \\
\hline$<\mathrm{ng}>$ & 2 & $\mathbf{1 9}$ & 90 \\
\hline$<\mathrm{mb}>$ & 2 & $\mathbf{1 1}$ & 85 \\
\hline$<\mathrm{nd}>$ & 1 & $\mathbf{6}$ & 86 \\
\hline$<\mathrm{nc}>,<\mathrm{nqu}>$ & $\mathbf{2 0}$ & 3 & 83 \\
\hline$<\mathrm{mp}>$ & $\mathbf{9}$ & 1 & 90 \\
\hline$<\mathrm{nt}>$ & $\mathbf{7}$ & 5 & 58 \\
\hline
\end{tabular}

Tabla 7. Resumen del análisis comparativo entre DST y quechua cuzqueño $(\mathrm{n}=86)$; correspondencia general: $84 \%(82 / 86)$. 


\begin{tabular}{|l|l|l|l|}
\hline Definición DST & Palabra DST & \multicolumn{1}{c|}{$\begin{array}{c}\text { Quechua Cuzco mod. } \\
\text { (e histórico GH) }\end{array}$} & Quichua ec \\
\hline cobre & anta & anta (anta) & NA \\
\hline oluidar & conca & qonqa (concca, cconcca, conca) & kunga \\
\hline adolescer, enfermar & huncu & onqoy (vncco, vnco) & ungu \\
\hline encontrar & tinco & tinku (tincu) & tingu \\
\hline llano & pampa & panpa (pamppa) & pamba \\
\hline errado & panta & panta (panta) & panda \\
\hline pescar peces con anzuelo & pinta & pinta (pinta) & NA \\
\hline caña & pinto & pintu (pintok) & NA \\
\hline juntar & tanta & tanta (tanta) & tanda \\
\hline
\end{tabular}

Tabla 8. Palabras con <ptc $>$ posnasal en DST sin oclusivas complejas en el quechua cuzqueño moderno $(\mathrm{n}=9)$.

DST y quechuas modernos es mínima y comparable a la variación dialectal actual (véase tabla 10, abajo).

Aunque no tienen una presencia estadísticamente significativa, hay que notar que existen nueve excepciones a las correspondencias de oclusivas simples con $<\mathrm{bdg}>$ y cinco a las de oclusivas complejas con $<$ ptc $>$. Sin embargo, estas tal vez no sean tan excepcionales en verdad, porque se agrupan en dos conjuntos que se definen por sus propiedades lingüísticas. En el primer conjunto, de palabras que tienen oclusivas sencillas modernas y que se escriben con $<$ ptc $>$ en DST, todas las palabras tienen oclusivas en ambas sílabas, excepto en un caso de una vocal inicial (<anta> para 'cobre', con sonorización en topónimos como Andamarca).

En la actualidad existe una restricción en palabras como estas, con oclusivas al inicio de ambas sílabas, donde no puede ocurrir una oclusiva compleja en la segunda sílaba. Sin embargo, es posible que esta restricción no se haya aplicado tan estrictamente en el pasado, porque vemos en algunos casos en la obra de $\mathrm{GH}$ el uso de dos grafemas dobles por palabra $(<$ ttantta $>,<$ ttimppu $>,<$ ttamppa $>$, etc.); mientras que en aimara, donde no existe la misma restricción, tenemos ejemplos modernos como /p'amp'ana/ (Lucca, 1983, p. 357).

También existe un conjunto de cinco excepciones a las tendencias estadísticas que van en la otra dirección, con oclusivas complejas en el cuzqueño-boliviano moderno, pero escritas con $<$ bdg $>$ por DST. Estas también forman una clase fonológica consistente: todas tienen fricativas en la primera posición, y oclusivas complejas en la segunda, aparte de una que inicia con vocal. A diferencia de las palabras con dos oclusivas, las palabras con una fricativa seguida por una oclusiva sí permiten la presencia de oclusivas complejas en el quechua cuzqueño-boliviano moderno.

Como los conjuntos de palabras en las tablas 8 y 9 tienen aspectos en común entre ellos, es posible que la variabilidad menor vista en los textos de DST con respeto al quechua cuzqueño moderno tenga una motivación histórica regular sobre la base de cambios en las restricciones de coocurrencia. De todas formas, como fue mencionado arriba, dada la variabilidad vista entre los dialectos del quechua cuzqueño-

\begin{tabular}{|l|l|l|l|}
\hline Definición DST & Palabra DST & $\begin{array}{c}\text { Quechua Cuzco mod. } \\
\text { (e histórico GH) }\end{array}$ & Quichua ec \\
\hline artesa & angara & anq'ara (ancara, anccara) & NA \\
\hline sudar & humbi & hunp'i (hunpi, hunppi) & humbi \\
\hline Ilenar & honda & hunt'a (hunta, huntta) & hunda \\
\hline trença & simba & sinp'a (cimpa, cimppa) & NA (shamba) \\
\hline barba & çunga, çumga & sunkha (çunca, çuncca) & sunga \\
\hline
\end{tabular}

Tabla 9. Palabras con sonorización en DST y oclusivas complejas en el quechua cuzqueño moderno $(\mathrm{n}=5)$. 


\begin{tabular}{|c|c|c|c|c|}
\hline \# Consistente & Bolivia & Ecuador & DST <ptc > vs. <bdg> & GH $<p p><t t><c h h\rangle$ \\
\hline Complejo & 93 & 25 & 16 & 66 \\
\hline Simple & 148 & 72 & 20 & 78 \\
\hline Inconsistente & 20 & 12 & 8 & 56 \\
\hline TOTAL & $n=261$ & $n=109$ & $n=44$ & $n=200$ \\
\hline$\%$ & 92 & 89 & 82 & 72 \\
\hline
\end{tabular}

Tabla 10. Comparación de tazas de consistencia de correspondencia de contraste sencillocomplejo del quechua cuzqueño (Cusihuamán, 1979; Gobierno Regional Cusco, 2005) con el quechua boliviano (Laime Ajacopa, et ál., 1997), el quichua ecuatoriano serrano (datos primarios, dialecto Cotopaxi), DST (indicado por sonorización) y GH (indicado por duplicación consistente de letras, o variación entre una y dos letras), con base en una lista estandarizada.

boliviano hoy con respeto a estos sonidos, este tipo de variación en un número pequeño de palabras no sorprende.

Finalmente, podemos preguntar si la variación con respeto a cuáles lexemas específicos tienen oclusivas complejas en diferentes quechuas tiene alguna implicación para los resultados que indique una correlación con el quechua cuzqueño (Cusihuamán, 1976; fuentes específicas: Gobierno Regional Cusco, 2005). Para explorar esta pregunta, se realizó una comparación de tres quechuas de tres países distintos: el cuzqueño, el boliviano y el ecuatoriano serrano. La tabla 10 ilustra las tasas de correspondencias del cuzqueño con estos otros dos quechuas comparados con las de la documentación colonial de DST y GH con base en una lista léxica comparativa estandarizada. Siguiendo a Dockum y Bowern (2019), utilizamos una lista estandarizada de 400 palabras ${ }^{10}$ como cantidad recomendada para una representación fonológica adecuada.

La variabilidad de las oclusivas complejas en diferentes quechuas a veces ha sido entendida como una irregularidad proveniente de una innovación fonológica instigada por contactolingüístico, pero aquí, en cambio, vemos grados altos de correspondencia más característicos de la retención que la innovación. Aunque en el quichua ecuatoriano solamente se puede comparar sonidos en las consonantes iniciales, porque no se preservan los reflejos en otras posiciones, la correspondencia de palabras con oclusivas complejas en quechua cuzqueño con esta lengua distante es casi tan alta como la del cuzqueño con el quechua más cercano, el boliviano, a pesar de también haber sido caracterizado como irregular y así descartado como un supuesto efecto "superestratístico" (Torero, 1984) o hasta "postizo" (Cerrón-Palomino, 2003, p. 187)11. El motivo de la propuesta del efecto "superestrato" es defender la agrupación "IIB" de quechua, ya que supuestamente el ancestro común del quichua ecuatoriano y el quechua de DST no tenía oclusivas complejas. Si ambos tenían estos sonidos, no habría mucha razón para recurrir a efectos de "superestrato" para explicar su presencia en Ecuador.

Para DST, se comparó basándose únicamente en palabras con oclusivas posnasales, porque solamente estas tienen evidencia del contraste sencillocomplejo (véase abajo en tabla 11 para análisis de duplicación de letras en DST). Entre el quechua de DST y el cuzqueño existe una correspondencia alta $(82 \%)$ similar al resultado reportado arriba basado en la obra completa de DST (84\%). El uso de duplicación por GH para escribir /q/ interfiere con la comparación de $<\mathrm{cc}>\mathrm{y}<\mathrm{qq}>$, pero si evaluamos solamente las otras consonantes que aparecen en forma duplicada $<\mathrm{pp}><\mathrm{tt}>$ y $<\mathrm{chh}>$ (consistentemente o como variante), encontramos una correspondencia mediana $(72 \%$; cfr. tabla 11 para detalles sobre <cc $>$ y $<q q>)$. Aunque GH representaba el quechua cuzqueño antiguo, su ortografía es menos consistente con el cuzqueño moderno que los otros quechuas comparados en tabla 10, algo que parece reflejar su dificultad en escribir los sonidos más que variabilidad histórica en pronunciaciones, dado la consistencia mucho mayor ente los tres quechuas modernos. Las correspondencias altas entre estos sugieren que la variación, aunque es un fenómeno real, es menos significante de lo que se pensaba, y este factor no alteraría los resultados cuantitativos aquí. 


\section{Discusión: contexto histórico ortográfico}

Recordemos que los dos libros clásicos de DST son los únicos ejemplos sustanciales del quechua del período ${ }^{12}$, y que la idea de que no representan oclusivas complejas se basa solamente en la ausencia de prácticas de escribirlas. Sin embargo, ¿es en verdad históricamente realista esperar que DST representara oclusivas complejas quechuas ortográficamente si ningún otro autor logró hacer esto durante todo el siglo XVI, hasta décadas después de su fallecimiento? Existen otros ejemplos escasos de quechua de mediados del siglo XVI, y, tal como la obra de DST, algunos de estos también utilizan los caracteres $<$ bdg $>$ y tampoco marcan oclusivas complejas (CerrónPalomino, 2017). Una revisión de estos fragmentos encontrará pocos contraejemplos a los resultados aquí, porque la gran mayoría de palabras con $<$ bdg $>$ posnasal ahí también tiene oclusivas simples en el quechua cuzqueño, precisamente como en la escritura de DST. Aceptando que la sonorización en el quechua de DST refleja la presencia de oclusivas complejas, ¿por qué no las escribió? En esta sección daremos contexto histórico que confirma que, por no escribir las oclusivas complejas, DST estaba siguiendo un patrón común visto en toda la documentación española temprana de las lenguas de las Américas.

Cuando se trata de la representación fonética colonial temprana existen dos tendencias generales: la primera es que cuando se escribía un sonido que sí existía en español, el mismo se representaba con relativa escrupulosidad con las mismas letras que se usaban en español, algo que parece aplicable al uso de $<$ bdg $>$ versus $<$ ptc $>$ en la obra de DST. La segunda tendencia es que cuando escribían palabras con sonidos que no existían en español, no se representaban estos sonidos regularmente en la ortografía inicial, y generalmente se tardaba décadas, si no siglos, para desarrollar formas de escribirlos, como en el caso de la africada retrofleja quechua $/ \hat{\mathrm{c}} /(<\mathrm{tr}>)$ que solo fue registrada en el siglo $\mathrm{XX}$ (Anónimo, 1905; cfr. Carreño, 2011, p. 14). Este proceso se repite una y otra vez en la documentación colonial: inicialmente no existen convenciones ortográficas para sonidos nuevos o son inconsistentes, y poco a poco, con tiempo, empiezan a escribir caracteres nuevos más consistentemente (Calvo Pérez, 2005), muchas veces eventualmente llegando a sistemas relativamente buenos para la época (SmithStark, 2005), pero nunca de forma inmediata sin un período de aprendizaje.

Esta etapa inicial empezó más temprano en Mesoamérica que en los Andes, por la anterioridad de la llegada de los españoles. Para el p'urhépecha, por ejemplo, por los primeros 50 años después de contacto se distinguen solamente $/ \mathrm{t}^{\mathrm{h}} /$, hasta que textos más tardíos empiezan a distinguir $/ \mathrm{p}^{\mathrm{h}} / \mathrm{y} /$ $\mathrm{k}^{\mathrm{h}}$ / (Monzón, 2005). Para las lenguas mayas, aunque la documentación muy temprana no representa las eyectivas (por ejemplo: Anónimo, 1547, en tzoztil), hubo la gran ventaja de la temprana contribución del alfabeto de Francisco de la Parra, quien inventó caracteres nuevos para estos sonidos alrededor del año 1550 (véase Smith-Stark, 2005, p. 17), algo que fue aplicado a otras lenguas de la región, pero muchas veces de forma irregular, como en documentos de xinca del siglo XVIII que solamente marcan $/ \mathrm{k}^{\prime} / \mathrm{y}$ no /p'/ o /t'/ (Maldonado de Matos, 1770; comparar con Rogers, 2010; Sachse, 2010). Hasta el siglo XX, todavía se registraban las consonantes glotalizadas mayas de forma inconsistente, como en el caso de Chicomuceltec (Norcliffe, 2003, p. 14).

Los avances ortográficos en Mesoamérica no llegaron a tener mucha influencia en Sudamérica, y en varias regiones aun hasta los siglos XVIII y XIX vemos gran cantidad de documentos donde no se escriben oclusivas complejas para lenguas que sí las tienen. En la Amazonía podemos citar fuentes coloniales tardías de la familia sáliba-piaroa que no registraban estos sonidos (Anónimo, 1790), aunque es probable que existían (Labrada, 2016, p. 203), además de muchos vocabularios recolectados durante el siglo XIX que no notaron estos tipos de contrastes para lenguas donde después estarían descritos, incluyendo koreguaje (tucana; Vergara y Vergara \& Delgado, 1860), tariana (arawaka; Coudreau, 1887) o tinigua (tinigua-pamigua; Ernst, 1891; Ortiz, 2000). En el Chaco hay muchas lenguas con consonantes eyectivas y todavía no se registraban en documentos del siglo XX, por ejemplo el chorote (mataco-guaicurú; Hunt, 1915; comparar con Carol, 2014). En la Patagonia tenemos varios ejemplos más de vocabularios tempranos que no distinguieron las oclusivas complejas, como por ejemplo el caso del selk'nam (Rojas Berscia, 2014, p. 25). 
Para los Andes, existen ejemplos como el chipaya, cuyas aspiradas y eyectivas también fueron escritas de forma irregular en las fuentes iniciales (Hannss, 2008, p. 65); estas tampoco fueron registradas inicialmente para el leko (Kerke, 2009). Para el aimara se mencionan las oclusivas complejas por primera vez en los textos del Tercer Concilio Limense de 1584, pero no se representan ortográficamente ahí ni en los primeros textos aimaras de Oré (1598). No es hasta los trabajos de Bertonio (1612a, 1612b y otros) donde se aplican caracteres especiales para estos sonidos, aunque así como en su Arte quechua, en su Arte aimara Torres Rubio (1616) no los aplica. Como Parra para las lenguas mayas, quien dio un modelo para otros autores, se puede observar que Bertonio fue el autor clave para aimara, y González Holguín para quechua, pero, de estos dos, solamente Bertonio logró distinguir eyectivas (letras duplicadas) de aspiradas $(\operatorname{con}<\mathrm{h}>$ ). La estrategia de González Holguín fue de escribir ambas con letras duplicadas, además de distinguir la /q/ de la misma forma (en adición a usos esporádicos de $<\mathrm{k}>$ ). La tabla 11 compara la consistencia ortográfica de $\mathrm{GH}$ para diferentes sonidos, y también enumera los usos de grafemas dobles en DST.

Aunque su ortografía es muy inconsistente, hay evidencia de que GH estaba intentando escribir tanto oclusivas complejas como consonantes uvulares con doble letras porque estas registran correspondencias más altas, mientras que para las oclusivas simples, menos /q/, las correspondencias son más bajas.
Como el uso de duplicación de letras de DST es infrecuente, la muestra léxica no identificó suficientes ejemplos para análisis, entonces se consideró todos los casos de duplicación de consonantes en su obra en la tabla 11, donde vemos que hay algo de evidencia de que DST usaba letras dobles para representar /q/ (apoyando el análisis de Cerrón-Palomino de evidencia vocálica; 1990, pp. 364-369), pero esto no parece haber sido una estrategia para escribir oclusivas complejas (véase Cerrón-Palomino, 1990, pp. 345-346).

Igualmente, en los otros textos del siglo XVI como del Tercer Concilio (1584) o el Symbolo Cathólico Indiano de Oré (1598; véase también AndrangoWalker, 2018), DST no usaba letras dobles para representar las oclusivas complejas, y algunas fuentes de inicios del siglo XVII como Martínez (1604) tampoco lo hacen, aunque Oré ya había empezado a escribir doble letras para esta época en su Rituale seu manuale Peruanum de 1607 (véase Durston, 2003, p. 199). Después de la publicación del Arte (1607) y Vocabulario (1608) de GH, con extensos ejemplos de su ortografía, esta todavía no fue aplicada en textos posteriores como Torres Rubio (1619), Pérez Bocanegra (1631) y Prado (1650 [1640]) o Molina (en Romero, 1928 [1649]; selecciones en Taylor, 2001), y solo sería con la llegada de una generación de autores todos nacidos en Perú como Ávila (1648), Avendaño (1649), Roxo Mexía (1648), y Bellarmino y Jurado Palomino (1649) que la norma de duplicación de letras se generalizaría más.

\begin{tabular}{|c|c|c|c|c|c|c|c|c|c|c|c|c|c|c|c|}
\hline GH & $\mathbf{q}$ & $\mathbf{q}^{\mathrm{h}}$ & $q^{\prime}$ & k & $\mathbf{k}^{\mathbf{h}}$ & $\mathrm{k}^{\prime}$ & $\mathbf{p}$ & $\mathbf{p}^{\mathbf{h}}$ & p' & $\mathbf{t}$ & $\mathbf{t}^{\mathrm{h}}$ & $\mathbf{t}^{\prime}$ & ch & $\operatorname{ch}^{h}$ & ch' \\
\hline$<\mathrm{C}>$ & 7 & 1 & 1 & 27 & 2 & 2 & 34 & 1 & 1 & 7 & 1 & 0 & 10 & 0 & 1 \\
\hline$<\mathrm{CC}>$ & 30 & 9 & 13 & 23 & 5 & 12 & 7 & 9 & 10 & 11 & 2 & 12 & 7 & 2 & 14 \\
\hline$\%$ & 81 & 90 & 93 & 46 & 71 & 86 & 17 & 90 & 91 & 61 & 67 & 100 & 41 & 100 & 93 \\
\hline DST & $\mathbf{q}$ & $\mathbf{q}^{\mathrm{h}}$ & $\mathbf{q}^{\prime}$ & k & $\mathbf{k}^{\mathbf{h}}$ & $k^{\prime}$ & $\mathbf{p}$ & $\mathbf{p}^{\mathbf{h}}$ & $\mathbf{p}^{\prime}$ & $\mathbf{t}$ & $\mathbf{t}^{\text {h }}$ & $\mathbf{t}^{\prime}$ & ch & $\operatorname{ch}^{h}$ & ch' \\
\hline$<\mathrm{CC}>$ & 9 & 9 & 7 & 5 & 0 & 2 & 4 & 0 & 0 & 1 & 1 & 3 & NA & NA & NA \\
\hline
\end{tabular}

Tabla 11. Correspondencias de duplicación de letras en GH, sobre la base de una lista comparativa estandarizada de 400 palabras; para DST, el conjunto completo de palabras con doble letras. 


\section{El libro Sermones de los Misterios de Nuestra Santa} $\mathrm{Fe}$ Católica en la Lengua Castellana y la General del Inca (terminado en 1648, publicado en 1649) de Avendaño es una fuente importante para comparar con los textos de DST porque en su prólogo dice que representa el quechua local y no de Cuzco, pero de todas formas escribe las oclusivas complejas con letras dobles como lo hace GH. Ha sido difícil reconciliar esta evidencia con la idea de que el quechua de Lima no tenía estos sonidos, y hasta se ha propuesto que "los censores" agregaron estas letras (Cerrón-Palomino, 2003, p. 120; Torero, 1972, p. 91) ${ }^{13}$. Esta propuesta ignora que toda la censura colonial se centraba en cuestiones de doctrina religiosa e herejía (Guibovich, 2001, p. 219; 2003; 2017), no de ortografía, y también ignora las palabras de los mismos censores incluidas en su aprobación inicial de la obra de Avendaño, que la califican así: "la ortografía de la lengua, genuina, y la mejor de quantas hasta oy se an escrito" (Avendaño, 1649; cfr. también Itier, 2000, pp. 51-52, 58). La presencia de las oclusivas complejas en el quechua de Lima también es mencionada por otra obra contemporánea, el Arte de Roxo Mexía y Ocón, quien nota en su introducción ${ }^{14}$ :

Pues no solo en la cortesana del Cuzco, a Buenos Ayres, sino en la inculta de por aca, sus vocablos, que son vnos en todo Perú, desde Quito a Buenos Ayres, tienen la misma variación por la pronunciación. Pues en todo el Reyno, distinguen con ella a Tuta. Por la Noche. Y Thutta. Por la Polilla. A Ccacca. Por la Peña, o Caca. Por el Tio. O Kcaça. El entonado. Y assi en todos los Vnisonos. (1648)

Esta observación concuerda con los altos grados de correspondencia vistos hasta hoy entre Cuzco, Bolivia y Ecuador tabla 10, arriba), y el uso de "por aca" refiere a Lima. Comparado a Avendaño, quien era nativo de Lima, DST había llegado a Perú a los 40 años de edad y parece haber sufrido de algún grado de dificultad de percepción de los contrastes fonémicos nuevos ("sound blindness"; véase Boas, 1889). Esto es precisamente el efecto que describe Garcilaso de la Vega para "un religioso dominico que en el Perú había sido cuatro años catedrático de la lengua general" que no escuchaba la diferencia entre /pača/ versus / $\mathrm{p}^{\mathrm{h}} \mathrm{ača} /\left(2016\right.$ [1609], p. 65) ${ }^{15}$. Al final, el contexto histórico colonial indica que los primeros autores no tienden a distinguir oclusivas complejas muy consistentemente, y entonces es muy normal que DST no las escribiera, y más bien sería algo sorprendente si lo hubiera logrado.

En contraste con Perú, en Ecuador las aspiradas contrastivas no se registran en casi ninguna fuente de la época colonial, y hasta ahora no se representan en la ortografía estandarizada. Solamente una fuente colonial de mitades del siglo XVIII escribe oclusivas complejas (el arte anónimo de Bogotá; DedenbachSalazar Sáenz, 1993; Garcés, 1999), mientras que las principales fuentes del siglo XIX no las escriben (Cordero, 1992 [1892]; Grimm, 1896). Es solamente en el siglo XX que empiezan a ser registradas de nuevo en Ecuador por estudios lingüísticos modernos. Aunque desde la perspectiva de los Andes sureños, donde estos sonidos han sido escritos por siglos, tal vez parece extraño que no se escriban, el caso del Ecuador es un ejemplo contemporáneo de cómo pueden existir oclusivas complejas quechuas en el habla sin ser representadas regularmente en la ortografía, así como parece haber sido el caso con DST históricamente.

\section{Conclusiones}

¿Se puede explicar la evidencia presentada arriba de otra forma que no sea proponer la existencia de consonantes complejas contrastivas en el quechua de DST? A pesar de la fuerte evidencia cuantitativa para este resultado, es probable que no sea aceptado por algunos investigadores que se subscriben religiosamente a la clasificación tradicional de las lenguas quechuas, ya que esta asume que tanto las oclusivas complejas como la sonorización son innovaciones en ramas independientes, totalmente sin conexión la una con la otra (Torero, 1964, pp. 463-465; Parker, 1963, pp. 248-249). La realidad de que podrían ser retenciones más antiguas, y además reflejos uno del otro, es un poco sorprendente, pero, si no hubo oclusivas complejas en el quechua de DST, ¿de qué otra forma podríamos explicar la alternancia?

Se puede anticipar la propuesta de que el vocabulario en cuestión fue introducido por DST de otros quechuas, haciendo referencia a la idea de que la obra de DST es multidialectal, algo mencionado por Parker (1969, p. 152) y Cerrón-Palomino (1990). Sin embargo, no es tan fácil escapar de las consecuencias de los resultados. Aquí no se discute la posibilidad de que la obra de DST sea multidialectal, y se acepta 
que es posible que él haya incluido algunas palabras recolectadas en sus viajes y de personas de diferentes partes del Perú, pero esto en sí no puede explicar la evidencia estadística.

El problema básico con la posición según la cual todas las palabras relevantes provienen de otros quechuas es que asume equivocadamente que solamente hay que explicar la presencia de palabras sin sonorización como material externo y así, convenientemente, descontarlas. Lo que realmente hay que explicar es por qué estas palabras corresponden regularmente con las que tienen oclusivas complejas en el quechua cuzqueño-boliviano, mientras que las palabras con oclusivas simples cuzqueñas muestran sonorización.

No parece posible que DST haya usado un criterio fonológico de este tipo para seleccionar palabras, creando este efecto de alternancia artificialmente, y tampoco parece factible que DST haya reemplazado la mayoría de las palabras $(57 \%)$ con oclusivas después de $/ \mathrm{n} /$ con pronunciaciones de otros quechuas, dejando solamente una minoría de palabras $(43 \%)$ con la pronunciación local con /bdg/. Si esta variación fuera de tipo dialectal, esperaríamos que DST incluya varias versiones de cada palabra, como hace en otros casos cuando explica dos pronunciaciones (<hámuy $>$ vs. <xámuy $>$ para "venir") o dos sinónimos regionales léxicos (<nácani> versus < pistani> para "dessollar"), pero en cambio DST es muy consistente en su uso de $<$ ptc $>$ versus $<$ bdg $>$ de una manera que corresponde estadísticamente al quechua cuzqueño-boliviano actual. Al final, el argumento de las introducciones foráneas resulta ser circular, diciéndonos que tenemos que descontar esta fuerte evidencia de oclusivas complejas en la obra de DST porque ya sabemos que la obra de DST no tiene evidencia de oclusivas complejas.

El propósito de este estudio no ha sido interpretar la historia de las lenguas quechuas, sino de describir el sistema lingüístico representado por las prácticas ortográficas de DST, y solamente hay espacio para una corta reflexión histórica. Los resultados sugieren que se debe investigar más profundamente la posibilidad de que las oclusivas complejas sean una retención compartida entre el quechua cuzqueño, el quechua de DST, el quechua boliviano y el quichua ecuatoriano. Desafortunadamente, la pregunta de la antigüedad de las oclusivas complejas en las lenguas quechuas ha sido confundida con la pregunta de la relación quechuas-aimaras (por ejemplo, Torero, 2002, pp. 151-159), pero son asuntos separados, ya que estos sonidos son comunes en Sudamérica y fácilmente podrían haberse desarrollado independientemente. Aquí se pregunta solamente sobre el origen de las oclusivas complejas quechuas, sin decir nada sobre la relación quechua-aimara.

La propuesta del origen externo de las oclusivas complejas en quechua sufre del problema básico de proponer que los fonemas nuevos no entraron por préstamos léxicos aimaras, sino por una fonologización triple que afectó palabras quechuas ya existentes $\left(\mathrm{C}>\mathrm{C}, \mathrm{C}^{\mathrm{h}}, \mathrm{C}^{\prime}\right)$; sin embargo, no propone condiciones fonológicas para este cambio, como piden las normas de reconstrucción histórica (Cser, 2015; Garrett, 2015; Hamann, 2014; Hoenigswald, 1960; Jakobson, 1978 [1949]; Salmons y Honeybone, 2015). La posibilidad de que estos sonidos en el quechua de DST sean retenciones merece ser considerada, porque analizar el proceso en la otra dirección como un colapse $\left(\mathrm{C}, \mathrm{C}^{\mathrm{h}}, \mathrm{C}^{\prime}>\mathrm{G}\right)$ para los quechuas sin oclusivas complejas evitaría caer en estos problemas metodológicos.

La presencia de oclusivas complejas en el quichua ecuatoriano ha presentado un problema persistente para su ubicación dentro de la clasificación tradicional quechua, algo que Torero intentó resolver con la idea de influencia de un "superestrato" cuzqueño sobre un supuesto "substrato" de "quechua pre-Inca" en Ecuador, pero esta propuesta no ha sido aceptada como históricamente factible (Hartmann, 1979; Hocquenghem, 2012). Aquí vemos una instancia del patrón de recurrir repetitivamente a causas externas como explicación del origen de las oclusivas complejas para descartar la posibilidad de que sean originales del quechua: (a) mezcla "superestrato" en Ecuador, (b) mezcla "multidialectal" en DST, (c) mezcla dialectal ortográfica por "los censores" de Avendaño, y (d) mezcla con "préstamos fonémicos" inicialmente desde aimara. Todas estas propuestas de mezcla externa parecen no ser motivadas por evidencia lingüística, sino por el deseo de evitar reconstruir estos sonidos para la familia internamente según la norma del método comparativo.

Aunque la ausencia de oclusivas complejas en muchos quechuas ha sido citada como prueba de un origen externo, hay varias líneas de evidencia de que estos sonidos podrían haber existido más 
antiguamente en quechuas que no los tienen ahora (Proulx, 1974; Landerman, 2011; Itier, 2016) ${ }^{16}$. Vale la pena seguir explorando la posibilidad de que las aspiradas y eyectivas pudiesen haber sido mucho más generalizadas en tiempos anteriores, "desde Quito a Buenos Ayres" como dice Roxo Mexía, y se hayan ido perdiendo con el tiempo en la mayoría de lenguas de la familia. Acordémonos que pasó precisamente esto con el proto-indoeuropeo, y actualmente no existen contrastes de oclusivas complejas en muchas lenguas cuyo ancestro común las tenían, incluyendo a casi todas las lenguas de Europa (Beckwith, 2007; Bomhard, 1986; Elbourne, 1998). En la familia quechua misma, en sus múltiples difusiones a la Amazonía y a Colombia desde los Andes ecuatorianos, se han colapsado las oclusivas complejas y simples, y se puede ver este proceso todavía en operación en el quichua serrano moderno en ciertos casos donde estos sonidos han empezado a desaparecer en el habla de los jóvenes mientras los mayores todavía los pronuncian. La cercanía léxica del quichua argentino con el quechua boliviano indicia una relación cercana (Torero, 1972, pp. 78-79; también Grimes, 1985), entonces es posible que este quechua asimismo haya tenido oclusivas complejas históricamente, aunque esto es debatido (Granda, 2001).

Finalmente, es importante reconocer que además de la presencia de oclusivas complejas en el quechua de DST, los datos indican un sistema en que el contraste complejo-simple se alternaba con un contraste sorda-sonora después de /n/. Aunque generalmente considerada periférica a la fonología de las lenguas quechuas, la sonorización alofónica de oclusivas es en realidad un proceso que afecta diversos quechuas en casi todas las regiones, incluyendo el quechua de Tarma (Puente Baldoceda, 1977), el quechua de Conchucos (donde afecta solamente /q/; Hintz, 2006), el quechua de Chachapoyas (Taylor, 1979), y hasta en algunos casos limitados en quechuas sureños. Algunas investigaciones recientes encuentran tanto sonorización como oclusivas complejas en un dialecto del quechua cuzqueño de Raqch’i, donde se sonoriza solamente en palabras sin oclusivas complejas de una forma parecida al quechua de DST (Escobar Zapata, 2019). El quichua serrano ecuatoriano sería otro ejemplo más de oclusivas complejas y sonorización alofónica en el mismo quechua ${ }^{17}$. Al final, el quechua de DST no parece haber sido tan divergente frente a las lenguas quechuas modernas. Aunque no encontramos un sistema exactamente igual, localizamos muchos de sus elementos difundidos en las lenguas quechuas hoy ${ }^{18}$.

Además, si revisamos la literatura tipológica y descriptiva, hallamos amplios precedentes para este tipo de sistema en diferentes lenguas del mundo. En lenguas con oclusivas complejas y sonorización es muy común que las oclusivas complejas tengan alguna relación alofónica o de neutralización en ciertos contextos, especialmente después de sonidos nasales (Hamann y Downing, 2017), algo muy prominente en las lenguas bantú (Herbert, 1987; Vail, 1972), con interacciones complejas similares observadas en lenguas tan variadas como las tibeto-birmanas (Chirkova y Chen, 2013), el coreano (Sohn, 2001), el armenio (Schirru, 2012; Seyfarth y Garellek, 2018), y en algunas partes de América en lenguas como el p’urhépecha (Camacho Cervantes, 2018; Foster, 1969) y el a'ingae/cofán (Borman, 1962; Repetti-Ludlow et ál., 2019); además, el aimara chileno sonoriza solamente en la ausencia de oclusivas complejas (Poblete y Salas, 1997). Los resultados cuantitativos señalan que el sistema del quechua de DST probablemente tenía alternancias entre contrastes simple-compleja y sonora-sorda como han sido observados en lenguas como estas. Otra posibilidad es que había existido un sistema así históricamente, pero que en tiempos de DST ya se habían perdido las oclusivas complejas, dejando solamente el rastro del contraste sordo-sonoro después de /n/. En cualquiera de los casos, es necesario reconstruir las oclusivas complejas para este quechua con el fin de explicar la variación.

Por mucho tiempo, el misterio de la aparente irregularidad de la sonorización en el quechua de DST ha pasado sin resolverse. Aquí se ha demostrado por primera vez una relación cuantitativa altamente significativa que lo resuelve, y el apéndice incluye los datos completos para que este resultado pueda ser verificado y replicado independientemente, usando una simple aplicación del método comparativo. En realidad, la respuesta de la pregunta de la misteriosa alternancia ortográfica de DST entre $<$ ptc $>$ y $<$ bdg $>$ estuvo todo el tiempo visible en su obra, pero nunca fue descubierta hasta ahora porque se asumía que no había nada más que descubrir. 


\section{Apéndice}

\begin{tabular}{|c|c|c|c|c|c|}
\hline Significado & $\begin{array}{l}\text { Domingo de } \\
\text { Santo Tomás }\end{array}$ & $\begin{array}{l}\text { Quechua } \\
\text { cuzqueño } \\
\text { moderno } \\
\text { (AML) }\end{array}$ & $\begin{array}{l}\text { Q. cuzqueño } \\
\text { antiguo } \\
\text { (González } \\
\text { Holguín) }\end{array}$ & $\begin{array}{l}\text { Quichua } \\
\text { ecuatoriano } \\
\text { serrano } \\
\text { (Quito) }\end{array}$ & $\begin{array}{l}\text { Quechua } \\
\text { Chachapoyas- } \\
\text { Lamas } \\
\text { (Taylor, 1979) }\end{array}$ \\
\hline nieruo, o vena de animal & anco, hanco & hank'u & anccu, ancco & angu & angu \\
\hline atormecerse & $\begin{array}{l}\text { çaçunca-, } \\
\text { cuçunca-, } \\
\text { coconca- }\end{array}$ & susunka- & çuçunca- & NA & šušu- \\
\hline espesar & çanco- & sankhu- & çanccu- & NA & sangu- \\
\hline machucar & chanca- & chanqa- & $\begin{array}{l}\text { chamcca, } \\
\text { chancca }\end{array}$ & NA & NA \\
\hline oluidar & conca- & qonqa- & $\begin{array}{l}\text { concca-, } \\
\text { cconcca-, } \\
\text { conca- }\end{array}$ & kunga- & kunga- \\
\hline columpiar & guaylluncu & wayllunk'u & huaylluncu & NA & $\mathrm{NA}$ \\
\hline aduce o atambor & guancar & wank'ar & huancar & wankar & NA \\
\hline açucena & hamancáy & hamanq'ay & hamancay & NA & amanga, \\
\hline $\begin{array}{l}\text { coxo de pie, derrengar, } \\
\text { deslomar }\end{array}$ & hanca & Hank'a & hancca, hanca & hanga & NA \\
\hline boca arriba & $\begin{array}{l}\text { hancarca, } \\
\text { ancalla }\end{array}$ & $\begin{array}{l}\text { hanq'a } \\
\text { (hant'a) }\end{array}$ & hantta, hanta & NA & NA \\
\hline tostar & hamca- & hank'a- & $\begin{array}{l}\text { hamcca-, } \\
\text { hamca- }\end{array}$ & NA & $\mathrm{NA}$ \\
\hline $\begin{array}{l}\text { afeytarse los ojos o } \\
\text { alcoholarse }\end{array}$ & hincu- & NA & viucayrucu- (?) & NA & NA \\
\hline en puntillas & hinqui & hink'i & hinqqui, hinqui & NA & $\mathrm{NA}$ \\
\hline adolescer, enfermar & huncu- & onqo- & vncco-, vnco- & ungu & ungu \\
\hline cabrillas, constelacion & huncuy & NA & NA & NA & NA \\
\hline tentar, atentar tocando & Ilamca-, Ilanca- & Ilank’a- & $\begin{array}{l}\text { llamcca-, } \\
\text { llama-, Ilamca- }\end{array}$ & NA & NA \\
\hline zapato, o alparga & Ilanqui & NA & NA & NA & NA \\
\hline acicalar & Illonco- & Ilunk'u- & $\begin{array}{l}\text { llunccu-, } \\
\text { Huncu- }\end{array}$ & NA & $\mathrm{NA}$ \\
\hline $\begin{array}{l}\text { solar o alguna cosa, cosa } \\
\text { sola de assi, losado de } \\
\text { mazacote }\end{array}$ & Iluncu- & NA & NA & NA & NA \\
\hline empollarse el hueuo & manca- & NA & NA & NA & NA \\
\hline $\begin{array}{l}\text { remolino de viento } \\
\text { pequeño }\end{array}$ & pillonco & $\mathrm{NA}$ & $\begin{array}{l}\text { pilluncuy, } \\
\text { ppilluncuy }\end{array}$ & NA & $\mathrm{NA}$ \\
\hline verguença & pinca- & p'enqa- & $\begin{array}{l}\text { ppencca-, } \\
\text { ppenca-, } \\
\text { penca- }\end{array}$ & pinga & pinga \\
\hline cumbrera de casa & pinco & NA & pincu & NA & pingu \\
\hline abismo, agua sin ondon & poyunco & p'oyonqo & $\begin{array}{l}\text { ppuyunccu, } \\
\text { ppuyuncu, } \\
\text { puyuncu }\end{array}$ & NA & $\mathrm{NA}$ \\
\hline descuydo & ranca, ranco & $\begin{array}{l}\text { ranqha, } \\
\text { rankha }\end{array}$ & rancca & $\mathrm{NA}$ & $\mathrm{NA}$ \\
\hline empujar & tanca- & tanqa- & $\begin{array}{l}\text { ttancca-, } \\
\text { ttanca-, } \operatorname{tanca-}, \\
\text { tancca- }\end{array}$ & $\begin{array}{l}\text { tanga-, } \\
\text { thanga- }\end{array}$ & tanga- \\
\hline tauano & tancayllo & tankayllu & ttamcayllo & NA & NA \\
\hline papirotes dar & tinca- & t'inka- & ttinca- & NA & NA \\
\hline encontrar & tinco- & tinku- & tincu- & tingu- & tingu- \\
\hline apretar uno al otro & tinqui- & t’inki- $^{\prime}$ & $\begin{array}{l}\text { ttinqqui-, } \\
\text { ttinqui-, } \\
\text { tinqqui- }\end{array}$ & NA & $\mathrm{NA}$ \\
\hline
\end{tabular}




\begin{tabular}{|c|c|c|c|c|c|}
\hline Significado & $\begin{array}{l}\text { Domingo de Santo } \\
\text { Tomás }\end{array}$ & $\begin{array}{l}\text { Quechua } \\
\text { cuzqueño } \\
\text { moderno } \\
\text { (AML) }\end{array}$ & $\begin{array}{l}\text { Q. cuzqueño } \\
\text { antiguo (González } \\
\text { Holguín) }\end{array}$ & $\begin{array}{l}\text { Quichua } \\
\text { ecuatoriano } \\
\text { serrano (Quito) }\end{array}$ & $\begin{array}{l}\text { Quechua } \\
\text { Chachapoyas- } \\
\text { Lamas } \\
\text { (Taylor, 1979) }\end{array}$ \\
\hline apostentar & tynquirka & NA & $\mathrm{NA}$ & NA & NA \\
\hline abejera & urúncuy & $\mathrm{NA}$ & NA & NA & $\mathrm{NA}$ \\
\hline abejon, falsa abeja & acatanga & NA & NA & NA & $\mathrm{NA}$ \\
\hline aguila, aue conoscida & $\begin{array}{l}\text { anga, anca, } \\
\text { (ancca) }\end{array}$ & anka & anca, ancca & anga & anga \\
\hline artesa & angara & ankara & $\begin{array}{l}\text { ancara, } \\
\text { anccara }\end{array}$ & NA & NA \\
\hline azul color & angax & $\begin{array}{l}\text { anqas, } \\
\text { ankhas }\end{array}$ & ancas & NA & angaš \\
\hline assar & canga- & kanka- & canca- & NA & kanga- \\
\hline barba & çunga, çumga & sunkha & çuncca, çunca & NA & $\begin{array}{l}\text { sunka, sunga, } \\
\text { songa }\end{array}$ \\
\hline perder/desaparecer & chinga- & chinka- & $\begin{array}{l}\text { chinca-, } \\
\text { chincca- }\end{array}$ & činga- & činga- \\
\hline grama, herua & chinga, chinca & NA & NA & NA & NA \\
\hline diez & chunga & chunka & $\begin{array}{l}\text { chunca, } \\
\text { chuncca }\end{array}$ & čunga & trúnga \\
\hline naríz & cinga & senqa & cenca, cencca & singa & singa \\
\hline elada, o yelo & $\begin{array}{l}\text { chollungoy, } \\
\text { cholloncoy }\end{array}$ & chullunku & $\begin{array}{l}\text { chullunccuy, } \\
\text { chulluncuy }\end{array}$ & NA & $\mathrm{NA}$ \\
\hline rodilla & congor & qonqor & $\begin{array}{l}\text { concor, } \\
\text { cconcor }\end{array}$ & kunguri & kunguri \\
\hline cuello & conga & kunka & cunca, cuncca & kunga & kunga \\
\hline mil & guaranga & waranqa & $\begin{array}{l}\text { huaranca, } \\
\text { huarancca }\end{array}$ & waranga & (waranga) \\
\hline saco, costal & longo, rongo & $\mathrm{NA}$ & NA & NA & $\mathrm{NA}$ \\
\hline Inca & inga, ynga & inka & inca & inga, inka & inga \\
\hline grillo, specie de cigarra & chilli mango & NA & NA & NA & $\mathrm{NA}$ \\
\hline tigre & otorongo & $\begin{array}{l}\text { uturunku, } \\
\text { otorongo }\end{array}$ & vturuncu & NA & NA \\
\hline flauta & pingollo & pinkuyllu & pincullu & $\begin{array}{l}\text { pingu/u, } \\
\text { pinguzu }\end{array}$ & $\begin{array}{l}\text { pinguğu } \\
\text { (pantorilla) }\end{array}$ \\
\hline puerta & pongo & punku & puncu & pungu & pungu \\
\hline hinchar & pungui- & punki- & punqui- & pungi- & pungi-, punki- \\
\hline coraçón o entrañas & songo & sonqo & sonco, soncco & šungu & šungu \\
\hline garganta & tongorí & tonqor & $\begin{array}{l}\text { toncor, } \\
\text { tonccori }\end{array}$ & tunguri & $\begin{array}{l}\text { tongor, } \\
\text { tunkuru }\end{array}$ \\
\hline falso & yanga & yanqa & yanca, yancca & yanga & yanga, yanka \\
\hline hongo o xeta & callampa & k'allanpa & callampa & $\begin{array}{l}\text { ka^amba, } \\
\text { kazamba }\end{array}$ & $\begin{array}{l}\text { kallampa, } \\
\text { kağampa }\end{array}$ \\
\hline floxo, descuydado & çampa & sanp'a & $\begin{array}{l}\text { çamppa, } \\
\text { campa }\end{array}$ & NA & $\mathrm{NA}$ \\
\hline bordar o broslar o labrar & $\begin{array}{l}\text { compa-, } \\
\text { comba-, } \\
\text { campa- }\end{array}$ & NA & NA & NA & NA \\
\hline leonado color & chumpi & ch'unpi & $\begin{array}{l}\text { chhumpi, } \\
\text { chumpi }\end{array}$ & NA & NA \\
\hline Significado & $\begin{array}{l}\text { Domingo de } \\
\text { Santo Tomás }\end{array}$ & $\begin{array}{l}\text { Quechua } \\
\text { cuzqueño } \\
\text { moderno } \\
\text { (AML) }\end{array}$ & $\begin{array}{l}\text { Q. cuzqueño } \\
\text { antiguo } \\
\text { (González } \\
\text { Holguín) }\end{array}$ & $\begin{array}{l}\text { Quichua } \\
\text { ecuatoriano } \\
\text { serrano } \\
\text { (Quito) }\end{array}$ & $\begin{array}{l}\text { Quechua } \\
\text { Chachapoyas- } \\
\text { Lamas } \\
\text { (Taylor, 1979) }\end{array}$ \\
\hline torondon del cabeça & çumpo & NA & NA & NA & NA \\
\hline
\end{tabular}




\begin{tabular}{|c|c|c|c|c|c|}
\hline sapo, rana & hampato & hanp'atu & $\begin{array}{l}\text { hamppatu, } \\
\text { hampatu }\end{array}$ & hambatu & ambatu \\
\hline açadón o pala & Ilampa & NA & lanpa & NA & lampa \\
\hline color & limpi & Ilimp'i & Ilimppi, Ilimpi & NA & NA \\
\hline blando & Ilampo & llamp'u & $\begin{array}{l}\text { llamppu, } \\
\text { llampu }\end{array}$ & 3ambu & llambu, ğambu \\
\hline decaido, por el frio & ompo & umphuy & vmppu, vmpu, & NA & NA \\
\hline Ilano & pampa & panpa & $\begin{array}{l}\text { pampa, } \\
\text { pamppa }\end{array}$ & pamba & $\begin{array}{l}\text { pampa, } \\
\text { pamba }\end{array}$ \\
\hline andas & rampa & NA & NA & NA & NA \\
\hline barro o veruga & rimpicota & NA & NA & NA & NA \\
\hline desmelenado & tampa & t'anpa & $\begin{array}{l}\text { ttamppa, } \\
\text { ttampa, tampa }\end{array}$ & $\begin{array}{l}\text { tamba, } \\
\text { thamba }^{\text {hamb }}\end{array}$ & tamba \\
\hline hervir & timpo- & t'inpu- & $\begin{array}{l}\text { ttimpu-, } \\
\text { timpu- }\end{array}$ & $\begin{array}{l}\text { timbu-, } \\
t^{\text {himbu- }}\end{array}$ & timbu \\
\hline de todo & yumpay & NA & NA & NA & $\begin{array}{l}\text { yumpay, } \\
\text { yumbe }\end{array}$ \\
\hline anteayer & canimba & kayninpa & $\begin{array}{l}\text { ccanimpa, } \\
\text { canimpa }\end{array}$ & NA & NA \\
\hline porra & chambi & chanpi & $\begin{array}{l}\text { chhampi, } \\
\text { champi }\end{array}$ & NA & NA \\
\hline allende el rio & chimba & chinpa & chinpa, chimba & čimba & čimba \\
\hline arremangar & chimballi- & NA & chimpalli- & NA & $\mathrm{NA}$ \\
\hline vaso grande & chumba & chunpa & chumpa & NA & čumba \\
\hline faxa & chumbi & chunpi & chumpi & čumbi & čumbi \\
\hline bordar, poner orilla & cumbi- & qonpi- & $\begin{array}{l}\text { cumpi-, } \\
\text { ccumpi- }\end{array}$ & NA & $\mathrm{NA}$ \\
\hline desembarcar, balsa & $\begin{array}{l}\text { guambo-, } \\
\text { guamboro }\end{array}$ & wanpuru & $\begin{array}{l}\text { huampu-, } \\
\text { huampo- }\end{array}$ & wambu- & NA \\
\hline curar & hambi- & hanpi- & $\begin{array}{l}\text { hampi-, } \\
\text { hamppi- }\end{array}$ & hambi- & ambi- \\
\hline sudar & humbi- & hunp'i- & $\begin{array}{l}\text { hunpi-, } \\
\text { hunppi- }\end{array}$ & humbi- & umbi- \\
\hline alamo & rambra & NA & NA & NA & lamras \\
\hline trença & simba & sinp’a & cimpa, cimppa & NA & šimba \\
\hline caço de heirro, caçuela & tócoychimbo & NA & $\begin{array}{l}\text { ttocoychimpu, } \\
\text { tocoy chimpu }\end{array}$ & NA & NA \\
\hline desinfamar & tumba- & tunpa- & tumpa- & NA & tumba \\
\hline mazamorra & uimbilla & NA & NA & NA & $\mathrm{NA}$ \\
\hline cobre o alambre & anta & anta & anta, antta & anta & NA \\
\hline guirnalda & chanta & ch'anta & canta, chhanta & NA & NA \\
\hline leña & Ilamta & Ilamta & $\begin{array}{l}\text { llamtta, } \\
\text { vamtta. Ilamta }\end{array}$ & yanta, yanda & yamta \\
\hline Significado & $\begin{array}{l}\text { Domingo de } \\
\text { Santo Tomás }\end{array}$ & $\begin{array}{l}\text { Quechua } \\
\text { cuzqueño } \\
\text { moderno } \\
\text { (AML) }\end{array}$ & $\begin{array}{l}\text { Q. cuzqueño } \\
\text { antiguo } \\
\text { (González } \\
\text { Holguín) }\end{array}$ & $\begin{array}{l}\text { Quichua } \\
\text { ecuatoriano } \\
\text { serrano } \\
\text { (Quito) }\end{array}$ & $\begin{array}{l}\text { Quechua } \\
\text { Chachapoyas- } \\
\text { Lamas } \\
\text { (Taylor, 1979) }\end{array}$ \\
\hline sombra & llanto & llanthu & Ilantu, lantu, & $\begin{array}{l}\text { 3andu, } \\
\text { Mandu }\end{array}$ & gandu, zandu \\
\hline tela & manta & mant'a & mantta, manta & manta (sp?) & manta, manda \\
\hline errado & panta & panta & panta & panda & panda, panta \\
\hline mariposa & pilpinto & pillpinto & pillpinto & pilpintu & pilpintu \\
\hline pescar peces con anzuelo & pinta & pinta & pinta & NA & NA \\
\hline embolver & pinto- & p'into- & $\begin{array}{l}\text { ppintu-, pinttu-, } \\
\text { pintu- }\end{array}$ & $\begin{array}{l}\mathrm{p}^{\text {hindu-, }} \\
\text { sindu- }\end{array}$ & pindu-, pintu- \\
\hline caña & pinto & pintu & pintok & NA & pindu, pintu \\
\hline amontonar & suntu, sumtu & suntu & suntu, sunttu & NA & NA \\
\hline pan & tanta, tamta & t'anta & ttanta, ttanta & tanda & tanda \\
\hline
\end{tabular}




\begin{tabular}{|c|c|c|c|c|c|}
\hline juntar & tanta- & tanta- & tanta- & tanda- & tanda- \\
\hline condor & condor & kuntur & cuntur & kundur & kundur \\
\hline humear & conda, quonda & NA & NA & NA & NA \\
\hline Ilenar & honda & hunt'a & hunta, huntta & hunda & unda \\
\hline sol & indi, yndi, etc. & inti & inti & indi & inti, indi \\
\hline afeytarse con colorado & mandora & NA & mantur & NA & NA \\
\hline arrugar & $\begin{array}{l}\text { quincdi-, } \\
\text { quincti- }\end{array}$ & q'enti- & qquentin & NA & šinti- \\
\hline intercambiar & randi-, ra^di- & ranti- & ranti-, rantti- & randi- & randi- \\
\hline granizo & rundo, runto & runtu & runtu & rundu & ruru \\
\hline \multicolumn{6}{|l|}{ morfemas gramaticales } \\
\hline Significado & $\begin{array}{l}\text { Domingo de } \\
\text { Santo Tomás }\end{array}$ & $\begin{array}{l}\text { Quechua } \\
\text { cuzqueño } \\
\text { moderno } \\
\text { (AML) }\end{array}$ & $\begin{array}{l}\text { Q. cuzqueño } \\
\text { antiguo } \\
\text { (González } \\
\text { Holguín) }\end{array}$ & $\begin{array}{l}\text { Quichua } \\
\text { ecuatoriano } \\
\text { serrano } \\
\text { (Quito) }\end{array}$ & $\begin{array}{l}\text { Quechua } \\
\text { Chachapoyas- } \\
\text { Lamas } \\
\text { (Taylor, 1979) }\end{array}$ \\
\hline topic/focus (sufijo) & $\mathrm{ca}$ & qa & ca? & ka, ga & ka \\
\hline hasta (sufijo) & cama & kama & cama & kama, gama & kaman \\
\hline plural (sufijo) & & kuna & cuna & kuna, guna & sapa \\
\hline de cada uno (sufijo) & $\begin{array}{l}\text { yncanca, } \\
\text { incanca }\end{array}$ & NA & yncanca & NA & NA \\
\hline tercera futuro (sufijo) & nga & nqa & nca & nga & nka \\
\hline segunda singular (sufijo) & ngui & nki & nqui & ngi & nki, yki \\
\hline genitivo (sufijo) & $\mathrm{pa}$ & $\mathrm{pa}$ & $\mathrm{pa}$ & pa, ba & $\mathrm{pa}$ \\
\hline dativo (sufijo) & pac & paq & pac & pak, bak & pa \\
\hline aditivo (sufijo) & pas & pas & pas & paš, baš & piš \\
\hline locativo (sujifo) & pi & pi & pi & pi, bi & pi \\
\hline acusativo (sufijo) & ta, cta (suffix) & ta & ta, cta & ta, da & ta \\
\hline comitativo (sufijo) & ntin (suffix) & ntin & ntin & intin, indin & ntin \\
\hline ablativo (sufijo) & manta (suffix) & manta & manta & $\begin{array}{l}\text { manta, } \\
\text { manda }\end{array}$ & manta \\
\hline \multicolumn{6}{|l|}{ otras palabras con $\langle\mathrm{d}\rangle\langle\mathrm{b}\rangle$} \\
\hline Significado & $\begin{array}{l}\text { Domingo de } \\
\text { Santo Tomás }\end{array}$ & $\begin{array}{l}\text { Quechua } \\
\text { cuzqueño } \\
\text { moderno } \\
\text { (AML) }\end{array}$ & $\begin{array}{l}\text { Q. cuzqueño } \\
\text { antiguo } \\
\text { (González } \\
\text { Holguín) }\end{array}$ & $\begin{array}{l}\text { Quichua } \\
\text { ecuatoriano } \\
\text { serrano } \\
\text { (Quito) }\end{array}$ & $\begin{array}{l}\text { Quechua } \\
\text { Chachapoyas- } \\
\text { Lamas } \\
\text { (Taylor, 1979) }\end{array}$ \\
\hline hembra pequeña & $\begin{array}{l}\text { (guarme) } \\
\text { guambra }\end{array}$ & warma & huarma & wambra & $\begin{array}{l}\text { wamra, } \\
\text { wambra }\end{array}$ \\
\hline atinar & hambra & NA & NA & NA & NA \\
\hline alamo & rambra & $\begin{array}{l}\text { lanran, } \\
\text { ramran, } \\
\text { ranpras }\end{array}$ & ram ram & NA & lamras \\
\hline ciego & habra & NA & hapra & NA & NA \\
\hline baho & guabci, guapci & NA & huapci & NA & NA \\
\hline barba & çabra, çapra & NA & çapra & NA & $\begin{array}{l}\text { šapra (rayo del } \\
\text { sol) }\end{array}$ \\
\hline seja, pestaña & $\begin{array}{l}\text { quessibra, } \\
\text { quexibra }\end{array}$ & qhechiphra & $\begin{array}{l}\text { qquechipeha, } \\
\text { quesipra }\end{array}$ & NA & kišípra, kišibra \\
\hline mondar dientes & $\begin{array}{l}\text { catqui-, } \\
\text { cadqui- }\end{array}$ & NA & katqqui- & NA & NA \\
\hline dar pasos & tatqui-, tadqui- & NA & tatqui- & takti- & NA \\
\hline canal & vithco, vidco & NA & NA & NA & NA \\
\hline frente & catquid & NA & NA & NA & NA \\
\hline
\end{tabular}




\section{Notas}

1 La información del quichua ecuatoriano es de datos primarios, con los dialectos de Quito y Cotopaxi como bases; adicionalmente se consideran datos primarios de un corpus grande (100+ horas transcritas) de otros dialectos ecuatorianos y del ingano colombiano. Por ello agradecemos a las comunidades andinas que contribuyeron y por los consejos de colegas: G. Camacho Ríos, M. Bruil, E. Norcliffe, G. Gallagher, N. Emlen, J. Homan, J. Birchall, G. Ennis, K. Grzech, F. Julca Guerrero, L. Michael, C. Fernández-Salvador, F. Torreira, J. Hualde, M. Alemán, S. AnderBois, J. Stewart, J. Haviland, y E. Rosés Labrada.

También se ha consultado con Stark y Muysken (1977) y Carpenter (1981). La información de Chachapoyas-Lamas fue encontrada principalmente en Taylor (1979); a veces fue imposible confirmar la presencia o ausencia de una palabra. Para el quichua ecuatoriano serrano, es posible encontrar unas pocas palabras y morfemas sin sonorización posnasal, particularmente ejemplos de /t/, como /inti/ para "sol" o /-manta/ /-munta/ para "de/desde". Para Chachapoyas-Lamas, no se pudo encontrar información detallada sobre variación, pero la sonorización posnasal parece ser casi universal en estos quechuas también.

2 Datos del quechua cuzqueño de diccionarios modernos: A. M. de la Lengua (Gobierno Regional Cusco, 2005) y Cusihuamán (1976).

3 Como ya fue mencionado, en los pocos casos como 'chollungoy' y 'cholloncoy' en que DST escribe de dos maneras; casos como estos fueron incluidos como sonoras.

4 Para el quichua ecuatoriano se aplica una ortografía alofónica con caracteres prácticos en vez de API, para facilitar la comparación con las ortografías históricas y modernas de las fuentes del quechua cuzqueño. Se utilizan dígrafos <ch> para /t 5 , <sh> para $/ \int /$, y $<$ zh $>$ para el alófono alveopalatal fricativo $/ 3 /$ del lateral palatal $/ K /$. Se usa $<\mathrm{h}>$ para representar sonidos que varían entre $/ \mathrm{h} / \mathrm{y} / \mathrm{x} /, \mathrm{y}<\mathrm{f}>$ para un caso de $/ \phi /$. Para el quechua colonial y cuzqueño moderno se usa la ortografía de la fuente original.

5 El fenómeno de restricciones de coocurrencia de sonidos laringalizados aparece en lenguas diversas (MacEachern, 1999), y se mencionan en estudios indoeuropeos clásicos en la Ley de Grassmann (Grassmann, 1863).

6 En el caso de la raíz de 'flotar' o 'navegar en agua' y también de 'balsa' o 'barco', en algunos casos ha adquirido eyección en el quechua cuzqueño moderno, pero no parece haberla tenido históricamente (véase la forma moderna wanpuru); este caso fue contado como una correspondencia simple-sonora. En cambio, en casos como rampa para 'andas', cuando no fue posible encontrar la palabra en el quechua cuzqueñoboliviano, fue incluida en el cálculo.

7 El ejemplo de <mandora> para 'achiote' no aparece en fuentes modernas cuzqueñas, pero consta en González Holguín (1608), y se incluye aquí igualmente. Se usa todavía en el quichua amazónico y el Ingano colombiano.

8 Ejemplos: $\langle$ anco $\rangle /<$ hanco $\rangle$, <çaçunca $\rangle /\langle$ cuçunca $\rangle /\langle$ çoçonca $\rangle,\langle$ çanco $\rangle$, $\langle$ chanca $\rangle$, $<$ conca $>$, $<$ guaylluncu $>$, <guancar $\rangle$, <hamancáy $\rangle$, $<$ hanca $\rangle$, $<$ hancarca $>/<$ ancalla $\rangle$,

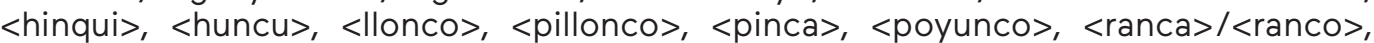

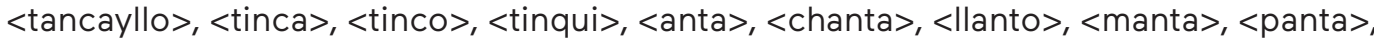
$<$ pilpinto $>$, <pinta $>$, <pinto $>$ ('embolver'), <pinto $>$ ('caña'), <tanta $>$.

9 Algunos dialectos modernos también registran variantes como sunka en vez de sunkha (Gobierno Regional Cusco, 2005, p. 252).

10 La lista comparativa combina la lista extendida Swadesh con 215 palabras (Swadesh, 1952, pp. 457-458), la lista Leipzig Jakarta (Haspelmath y Tadmor, 2009) y un conjunto de palabras adicionales con enfoque andino, por ejemplo con términos de flora y fauna local, con un total de 440 palabras, que permite alcanzar entre 300-400 cognados por cada par de lenguas quechuas comparadas. Se permite hasta dos sinónimos para cada término, y se escoge pares de cognados para comparación por sus sonidos relevantes (lexemas con oclusivas y africadas, solamente posnasales para DST).

11 La supuesta irregularidad de las oclusivas complejas ha sido el argumento principal por su caracterización como efectos "superestratístocos", pero aunque se pueden encontrar casos irregulares, en realidad las correspondencias generales son muy altas. El estudio de Muysken (2019, p. 103) no toma en cuenta que cada dialecto regional de 
quichua serrano tiene reflejos distintos de las oclusivas complejas antiguas, algunos colapsando las eyectivas y las aspiradas como aspiradas, otras veces convirtiendo las aspiradas en fricativas y colapsando las eyectivas con las oclusivas simples, y también cuenta palabras ecuatorianas con aspiración que no tienen cognados cuzqueños como no-correspondencias, presentando una correspondencia de $33 \%$; corregido por estos factores, sus datos tienen una taza de $70 \%$ para las 31 palabras mencionadas. Ello está más cerca a la figura real; se estima un promedio de $75 \%$ de correspondencia fonológica del conjunto total de palabras con aspiración en quichua con cognados comparables en el quechua cuzqueño (50+ palabras). Uno de los dialectos que más preserva estos sonidos es el de Cotopaxi, que fue utilizado para la comparación en tabla 10, que dio una correspondencia de $89 \%$.

12 También no nos olvidemos que la razón porque se cree que el quechua de DST fue tan diferente del quechua cuzqueño-boliviano es únicamente por sus supuestas diferencias fonológicas. Con respeto a la morfosintaxis, desde sus clasificaciones originales Parker observó para el quechua de DST que "naturalmente sospecharíamos que él había simplemente escrito el dialecto cuzqueño sin haberse preocupado en implementar la ortografía española para representar contrastes de tipos noespañoles" (Parker, 1969, pp. 166-167; traducción propia). Esta posibilidad parece ser cercana a lo que pasó en realidad; el quechua del DST parece haber tenido mucho más en común fonológicamente con el quechua cuzqueño de lo que se pensaba.

13 Torero (1972, p. 91) también menciona que solamente los diez primeros sermones usan doble letras, pero aparentemente no notó en el prólogo cuando señala que estos son los sermones del Tercer Concilio (1584) en su ortografía original (Itier, 2000, p. 58; Durston, 2007, p. 166); las doble letras deben ser la escritura de Avendaño mismo, porque de otra forma los censores las hubieron agregado a estos sermones también.

14 Aunque Durston describe fuertes rivalidades entre Avendaño y Roxo Mexía (2007, pp. 128-130), esto parece ser una especulación indirecta; de todas formas las discusiones de "Chinchaysuyo" quechua en estos textos seguramente tiene más que ver con sus divergencias léxicas y morfosintácticas que su ortografía.

15 Existen otras caracterizaciones de estos comentarios de Garcilaso de la Vega, que estaba "pecando de glotocentrismo" y no sabía que en otros quechuas aparte de su nativo cuzqueño no había contrastes de oclusivas complejas (Cerrón-Palomino, 2003, p. 346), pero no parece posible que un hablante nativo de quechua, y además un intelectual tan ilustre como Garcilaso de la Vega, pudiera haber viajado entre Cuzco y Lima en 1560 sin percibir las diferencias lingüísticas locales.

16 A esto podemos agregar la correspondencia en consonantes iniciales entre / ̌́c / en

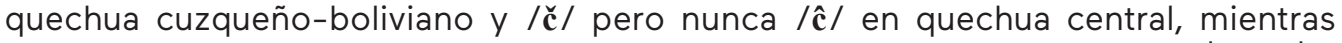

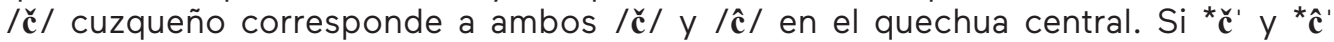
son los sonidos originales, se explica con un sencillo proceso de colapse /č́ /, / $\hat{c}^{\prime} /, / \check{c} />/ \check{c} /$, preservando el retroflejo original solamente en casos de oclusivas simples /ĉ/ en el quechua central. Palabras compartidas entre lenguas quechua y el jaqaru como /saĉ'a/ y /miĉ́a/ podrían preservar la /ĉ' / original pero solamente en posición intervocálica (véase Valverde, 2015a; 2015b). Analizar el proceso en la otra dirección, después del colapse /č/, /ĉ/ > /č/ en quechua sureño, nos obliga a proponer otra vez una fonologización sin motivación fonológica: /č/ > /č/ /č' /,

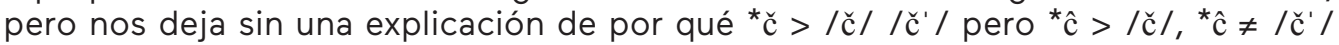
para el quechua cuzqueño-boliviano.

17 En Ecuador oclusivas complejas y sonorización ocurren en la misma palabra sin ninguna alternación o restricción, resultando en formas que no ocurrirían en el sistema específico de DST: /thamba/, /thimbu/, /phindu/, /thanda/, /phanga/, / chamba/ etc.

18 Adicionalmente, investigaciones recientes han mostrado que patrones acentuales pueden ser más diversos que un sistema simple de acento penúltimo, visto tanto en el uso del acento de DST como en algunos quechuas modernos como Chachapoyas (Valqui, 2018). 


\section{Referencias bibliográficas}

Andrango-Walker, C. (2018). El Símbolo católico indiano (1598) de Luis Jerónimo de Oré. Saberes coloniales y los problemas de la evangelización en la región andina. Iberoamericana, Vervuert.

Anónimo. (1547). Credo tzotzil. Manuscrito. John Carter Brown Library. https://archive.org/ details/credoindeumpatre00pabl

Anónimo. (1584). Doctrina cristiana y catecismo para la instrucción de los indios. Antonio Ricardo. https://archive.org/details/doctrinachristia00cath

Anónimo. (1586). Arte, y vocabulario en la lengua general del Peru llamada Quichua, y en la lengua española. Antonio Ricardo. http://archive.org/details/arteyvocabulario00unkn

Anónimo. (1790). Arte de la Lengua Saliba. San Miguel del Macuco: Manuscrito. https: / /bibliotecanacional.gov.co/es-co/colecciones/biblioteca-digital/ publicacion? nombre=Arte+de+la+lengua+saliva

Anónimo. (1905). Vocabulario políglota incaico: Comprende más de 12,000 voces castellanas y 100,000 de keshua del Cuzco, Ayacucho, Junín, Ancash y Aymará. Lima: Tipografía del Colegio de propaganda fide del Perú. http://archive.org/details/vocabulariopolg01unkngoog

Avendaño, F. de. (1649). Sermones de los misterios de nuestra santa fe catolica, en lengua castellana, y la general del Inca. Lima: Jorge Lopez de Herrera. http://archive.org/details/ sermonesdelosmis00aven

Ávila, F. de. (1648). Tratado de los evangelios. Imprenta de Pedro de Cabrera. https: //archive. org/details/tratadodeloseuan00avil

Beckwith, C. I. (2007). On the Proto-Indo-European Obstruent System. Historische Sprachforschung / Historical Linguistics, 120, 1-19. https://doi.org/10.13109/ hisp.2007.120.1.1

Bellarmino, R. F. R., y Jurado Palomino, B. (1649). Declaracion copiosa de las quatro partes mas essenciales, y necessarias de la doctrina christiana. Jorge López de Herrera. http:// archive.org/details/declaracioncopio00bell

Bertonio, L. (1612a). Arte de la lengua aymara, con vna silva de phrases dela misma lengua, y su declaracion en Romance. Juli: Francisco del Canto. http://archive.org/details/ artedelalenguaay00bert.

Bertonio, L. (1612b). Vocabulario de la lengua aymara. Juli: Francisco del Canto. http://archive. org/details/vocabulariodelalO0bert.

Betanzos, J. de. (1880 [1551]). Suma y narración de los Incas. Madrid: Manuel de Hernán-dez.

Boas, F. (1889). On Alternating Sounds. American Anthropologist, 2(1), 47-54. https://doi. org/10.1525/aa.1889.2.1.02a00040.

Bomhard, A. R. (1986). The Aspirated Stops of Proto-Indo-European. Diachronica, 3 (1), $67-$ 79. https://doi.org/10.1075/dia.3.1.05bom

Borman, M. B. (1962). Cofan phonemes. En B. Elson (Ed.), Studies in Ecuadorian Indian languages 1 (45-59). University of Oklahoma: Summer Institute of Linguistics.

Calvo Pérez, J. (2005). Fonología y ortografía de las lenguas indígenas de América del Sur a la luz de los primeros misioneros gramáticos. En O. Zwartjes \& C. Altman (Eds.), Missionary linguistics II. Lingüística misionera II. Orthography and phonology (pp. 137-170). Ámsterdam: John Benjamins Publishing Company. https://doi.org/10.1075/sihols.109.08cal

Camacho Cervantes, L. (2018). Purepecha Aspirated Consonants and Their Phonetic Variants. (Tesis de maestría). City University of New York. https://academicworks.cuny.edu/ gc_etds/2719

Carbajal Solís, V. C. (2004). Determinación de la frontera dialectal del quechua ayacu-chano y cuzqueño en el departamento de Apurímac. (Tesis de doctorado). Universi-dad Nacional Mayor de San Marcos, Lima, Perú. https://hdl.handle.net/20.500.12672/1227

Carol, J. (2014). Lengua chorote (mataguayo): Estudio fonológico y morfosintáctico. Lincom Europa.

Carpenter, L. K. (1982). Ecuadorian Quichua: Descriptive Sketch and Variation. (Tesis de Doctorado). University of Michigan, Ann Arbor, Estados Unidos de América.

Carreño, P. H. M. (2011). Las africadas del quechua central: Cronología de tres cambios lingüísticos. Sorda y Sonora, 1, 4-29. 
Cerrón-Palomino, R. (1990). Reconsideración del llamado "quechua costeño". Revista Andina, 16 (2), 335-409.

Cerrón-Palomino, R. (2003). Lingüística quechua (2. ${ }^{a}$ ed.). Centro de Estudios Regionales Andinos Bartolomé de Las Casas.

Cerrón-Palomino, R. (2017). El quechua "del ynga" según testimonio de los primeros cronistas. En J. C. Godenzzi y C. Garatea (Eds.), Literaturas orales y primeros textos coloniales. Volumen 1 (pp. 83-103). Fondo Editorial Pontificia Universidad Católi-ca del Perú.

Chirkova, K., y Chen, Y. (2013). Lizu. Journal of the International Phonetic Association, 43(1), 75-86. https://doi.org/10.1017/S0025100312000242

Cieza de León, P. de. (1553). Parte primera de la chronica del Peru. Sevilla: Martín de Montesdoca. http://archive.org/details/parteprimeradelaOOciez

Cordero, L. (1992 [1892]). Diccionario Quichua-Castellano y Castellano-Quichua (5. ${ }^{a}$ ed.). Corporación Editora Nacional.

Coudreau, H. A. (1887). La France équinoxiale. Tome Second: Voyage a travers les Guya-nes et I'Amazonie. París: Challamel aîné.

Cser, A. (2015). Basic Types of Phonological Change. En P. Honeybone y J. Salmons (Eds.), The Oxford Handbook of Historical Phonology (pp. 192-204). Oxford Uni-versity Press. https://doi.org/10.1093/oxfordhb/9780199232819.013.036

Cusihuamán, A. (1976). Diccionario quechua, Cuzco-Collao. Lima: Ministerio de Educa-ción.

Dedenbach-Salazar Sáenz, S. (1993). Una Gramática colonial del Quichua del Ecuador. Institute of Amerindian Studies, University of St. Andrews.

Dockum, R., y Bowern, C. (2019). Swadesh lists are not long enough: Drawing phonologi-cal generalizations from limited data. Lang. Document. Descript, 16, 35-54.

Durston, A. (2007). Pastoral Quechua: The History of Christian Translation in Colonial Peru, 1550-1650. University of Notre Dame Press.

Elbourne, P. (1998). Proto-Indo-European Voiceless Aspirates. Historische Spra-chforschung / Historical Linguistics, 111(1), 1-30.

Ernst, A. (1891). Ueber einige weniger bekannte Sprachen aus der Gegend des Meta und oberen Orinoco. Zeitschrift Für Ethnologie, 23, 1-13.

Escobar Zapata, E. (2019). Sobrevivencia del Quechua Inga en Raqch'i-Cusco. Tierra Nuestra, 13 (2), 12-26. https://doi.org/10.21704/rtn.v13i2.1400.

Ezcurra Rivero, Á., \& Bendezú-Araujo, R. (2017). Gramáticas y vocabularios coloniales del quechua y del aimara (1560-1619). En J. C. Godenzzi y C. Garatea (Eds.), Historia de las literaturas en el Perú. Volumen 1: Literaturas orales y primeros textos colonia-les (123-162). Fondo Editorial Pontificia Universidad Católica del Perú.

Fisher, R. A. (1922). On the Interpretation of $\chi 2$ from Contingency Tables, and the Calcula-tion of P. Journal of the Royal Statistical Society, 85(1), 87-94. https://doi.org/10.2307/2340521

Foster, M. L. (1969). The Tarascan Language. University of California.

Gallagher, G. (2013). Speaker awareness of non-local ejective phonotactics in Cochabamba Quechua. Natural Language \& Linguistic Theory, 31(4), 1067-1099. https://doi.org/10.1007/ s11049-013-9200-1

Garcés, F. (1999). Cuatro textos coloniales del quichua de la Provincia de Quito. Ministerio de Educación.

Garcilaso de la Vega, I. (2016 [1609]). Comentarios reales de los Incas. Universidad Inca Garcilaso de la Vega.

Garrett, A. (2015). Sound change. En C. Bowern y B. Evans (Eds.), The Routledge hand-book of historical linguistics (227-248). Routledge.

Gobierno Regional Cusco. (2005). Diccionario Qechua-Español-Quechua Qheswa-españolqheswa simi taqe (2. ${ }^{a}$ ed.). Gobierno Regional Cusco.

González Holguín, D. (1607). Gramatica y arte nueua de la lengua general de todo el Peru, llamada lengua Quichua, o lengua del Inca. Lima: Francisco del Canto. http://archive.org/ details/gramaticayartenu00gonz

González Holguín, D. (1608). Vocabulario de la lengua general de todo el Perú llamada lengua qquichua o del Inca. Imprenta Santa María.

Granda, G. de. (2001). Historia lingüística y tipología genética del quechua de Santiago del Estero, Argentina. Revista Andina, 33. 
Grassmann, H. (1863). Ueber die aspiraten und ihr gleichzeitiges vorhandensein im an- und auslaute der wurzeln. Zeitschrift Für Vergleichende Sprachforschung Auf Dem Ge-biete Des Deutschen, Griechischen Und Lateinischen, 12(2), 81-110.

Grimes, J. E. (1985). The Interpretation of Relationships among Quechua Dialects. Oceanic Linguistics Special Publications, 20, 271-284.

Grimm, J. M. (1896). La lengua quichua: (Dialecto de la República del Ecuador). Friburgo de Brisgovia: B. Herder.

Guibovich Pérez, P. (2001). Custodios de la ortodoxia: los calificadores de la Inquisición de Lima, 1570-1754. Revista de la Inquisición: (intolerancia y derechos humanos), 10, 213229.

Guibovich Pérez, P. (2003). Censura, libros e inquisición en el Perú colonial, 1570-1754. Sevilla: Universidad de Sevilla.

Guibovich Pérez, P. (2017). La censura de libros. En R. Chang-Rodríguez y C. García-Bedoya M. (Eds.), Historia de las Literaturas en el Perú, vol. 2: Literatura y cultura en el Virreinato del Perú: Apropiación y diferencia (pp. 57-84). Fondo Editorial de la Pontificia Universidad Católica del Perú.

Hamann, S. (2014). Phonological changes. En C. Bowern y B. Evans (Eds.), The Routledge Handbook of Historical Linguistics (pp. 249-263). Routledge.

Hamann, S., y Downing, L. J. (2017). *NT revisited again: An approach to postnasal laryn-geal alternations with perceptual Cue constraints. Journal of Linguistics, 53 (1), 85-112. https: // doi.org/10.1017/S0022226715000213.

Hannss, K. (2008). Uchumataqu: The Lost Language of the Urus of Bolivia ; a Grammatical Description of the Language as Documented Between 1894 and 1952. CNWS Publi-cations.

Hartmann, R. (1979). ¿ "Quechismo preincaico" en el Ecuador? Ibero-Amerikanisches Ar-chiv, 5 (3), 267-299.

Haspelmath, M., y Tadmor, U. (2009). Loanwords in the World's Languages: A Compara-tive Handbook. Mouton De Gruyter.

Herbert, R. K. (1987). Articulatory Modes and Typological Universals: The Puzzle of Bantu Ejectives and Aspirates. En R. Channon y L. Shockey (Eds.), In honor of Ilse Le-histe (pp. 401-413). De Gruyter Mouton. https://doi.org/10.1515/9783110886078.401

Hintz, D. M. (2006). Stress in South Conchucos Quechua: A Phonetic and Phonological Study. International Journal of American Linguistics, 72(4), 477-521. https://doi. org/10.1086/513058

Hocquenghem, A. M. (2012). How did Quechua reach Ecuador? En P. Heggarty y D. Ber-esfordJones (Eds.), Archaeology and Language in the Andes (pp. 345-373). British Academy.

Hoenigswald, H. M. (1960). Language Change and Linguistic Reconstruction. University of Chicago Press.

Hunt, R. J. (1915). El Choratio Yófuaha. Liverpool: Henry Young.

Itier, C. (1995). El teatro Quechua en el Cuzco. Institut Français d'Études Andines.

Itier, C. (2000). Lengua general y quechua cuzqueño en los siglos XVI y XVII. En Desde afuera y desde adentro. Ensayos de etnografía e historia del Cuzco y Apurímac (47-59). National Museum of Ethnology.

Itier, C. (2016). La formación del quechua ayacuchano, un proceso inca y colonial. Bulletin de I'Institut Français d'Études Andines, 45(2), 307-326. https://doi.org/10.4000/bifea.8003

Jakobson, R. (1978 [1949]). Principles of historical phonology. En P. Baldi y Werth, Ronald N. (Eds.), Readings in Historical Phonology: Chapters in the Theory of Sound Change (103120). University Park: Pennsylvania State University Press.

Kerke, S. van de. (2009). El Leko. En M. Crevels y P. Muysken (Eds.), Lenguas de Bolivia: Ambito Andino (287-332). Plural Editores.

Labrada, J. E. R. (2016). Proto-Sáliban Verb Classes. International Journal of American Linguistics, 82(2), 181-210. https://doi.org/10.1086/685901

Laime Ajacopa, T., Layme P, Félix, Plaza, P., y Cazazola, E. (1997). Diccionario bilingüe quechuacastellano, castellano-quechua. Centro Cultural JAYMA.

Landerman, P. (2011). Internal reconstruction in Aymara and Quechua. En J. H. Hill, L. Campbell, P. J. Mistry (Eds.), The Life of Language (35-58). De Gruyter Mouton. 
Lucca D., M. de. (1983). Diccionario aymara-castellano, castellano-aymara. Comisión de Alfabetización y Literatura en Aymara.

MacEachern, M. R. (1999). Laryngeal Cooccurrence Restrictions. Garland Press.

Maldonado de Matos, M. (1770). Arte de la lengua szinca: Con algunas reflexiones criticas a la arte K'akchiquel. Manuscrito.

Martínez, J. (1604). Vocabvlario de la lengva general del Perv llamado quichua [reedición de Tercer Concilio 1584]. Lima.

Monzón, C. (2005). Tarascan Orthography in the 16th Century: The Franciscan sources of inspiration and their analysis. En Missionary linguistics II. Lingüística misionera II. Orthography and phonology (pp. 65-88). John Benjamins.

Muysken, P. (2019). El kichwa ecuatoriano: Orígenes, riqueza, contactos. Quito: Abya-Yala.

Norcliffe, E. (2003). The reconstruction of Proto-Huastecan. (Tesis de Maestría). University of Canterbury, Christchurch, Nueva Zelanda. https://doi.org/10.26021/4646

Oré, L. J. de. (1598). Symbolo Cathólico Indiano. Lima: Antonio Ricardo. http://archive.org/ details/simbolo-catholico-indiano-peru

Ortiz, N. T. (2000). La Lengua Tinigua: Anotaciones fonológicas y morfológicas. En Len-guas indígenas de Colombia: Una visión descriptiva (pp. 669-679). Instituto Caro y Cuervo.

Parker, G. (1963). La clasificación genética de los dialectos quechuas. Revista del Museo Nacional, 32, 241-252.

Parker, G. (1969). Comparative Quechua Phonology and Grammar, IV: The Evolution of Quechua A. Working Papers in Linguistics [University of Hawaii], 1(9), 149-204.

Parker, S., y Weber, D. (1996). Glottalized and Aspirated Stops in Cuzco Quechua. International Journal of American Linguistics, 62(1), 70-85. https://doi.org/10.1086/466276

Pearson, K. (1900). On the criterion that a given system of deviations from the probable in the case of a correlated system of variables is such that it can be reasonably sup-posed to have arisen from random sampling. The London, Edinburgh, and Dublin Philosophical Magazine and Journal of Science, 50 (302), 157-175. https://doi.org/10.1080/14786440009463897

Pérez Bocanegra, J. (1631). Ritual formulario, e institucion de curas, para administrar a los naturales de este reyno, los santos sacramentos del baptismo, confirmacion, eucaris-tia, y viatico, penitencia, extremauncion, y matrimonio. Geronymo de Contreras. Re-cuperado de http://archive.org/details/ritualformulario00pr

Poblete, M. T. y Salas, A. (1997). El aymara de Chile (fonología, textos, léxico). Revista de Filología y Lingüística de la Universidad de Costa Rica, 23(121-203), 95-138. https://doi. org/10.15517/rfl.v23i1.20396

Prado, P. de. (1650). Directorio espiritual en la lengua española, y quichua general del inga. Compuesto por el padre Pablo de Prado, de la Compaña de lesus. Lima: Luis de Lyra. http://archive.org/details/directorioespiri00prad

Proulx, P. (1974). Certain Aspirated Stops in Quechua. International Journal of American Linguistics, 40 (3), 257-262. https://doi.org/10.1086/465319

Puente Baldoceda, B. (1977). Fonología del quechua tarmeño. Universidad Nacional Ma-yor de San Marcos, Centro de Investigación de Lingüística Aplicada.

Quiroga, P. de. (1992 [1569]). Coloquios de la verdad (D. Rípodas Ardanaz, Ed.). Instituto de Cooperación Iberoamericana, Casa-Museo de Colón, Seminario Americanista.

Repetti-Ludlow, C., Zhang, H., Lucitante, H., AnderBois, S., y Sanker, C. (2019). A'ingae (Cofán). Journal of the International Phonetic Association, 1-14. https://doi.org/10.1017/ S0025100319000082.

Rogers, C. (2010). A Comparative Grammar of Xinkan (Tesis Doctoral). University of Utah, Salt Lake City, Estados Unidos.

Rojas Berscia, L. M. (2014). A heritage reference grammar of Selk'nam (Tesis Doctoral). Radboud University Nijmegen, Nimega, Países Bajos. http://etnolinguistica.wdfiles.com/ local--files/tese\%3Arojas-berscia-2014/rojas_berscia_2014_selknam.pdf

Romero, C. A. (1928). Un libro interesante. Revista Histórica, 9 (1), 51-87.

Roxo Mexia y Ocón, J. (1648). Arte de la lengua general de los indios del Peru. Lima: Jorge López de Herrera. http://archive.org/details/artedelalenguage00roxo.

Sachse, F. (2010). Reconstructive Description of Eighteenth-century Xinka Grammar. LOT. 
Salmons, J., y Honeybone, P. (2015). Structuralist Historical Phonology (P. Honeybone y J. Salmons, Eds.). Oxford: Oxford University Press. https://doi.org/10.1093/ oxfordhb/9780199232819.013.029

Santo Tomás, D. de. (1560a). Grammatica, o Arte de la lengua general de los Indios de los reynos del Peru. Francisco Fernández de Córdova.

Santo Tomás, D. de. (1560b). Lexicon, o Vocabulario de la lengua general del Peru. Vallo-dolid: Francisco Fernández de Córdova. http://archive.org/details/lexiconovocabulaO0domi

Schirru, G. (2012). Laryngeal features of Armenian dialects. En B. Nielsen Whitehead, T. Oldander, y B. A. Olsen (Eds.), The sound of Indo-European: Phonetics, phone-mics, and morphophonemics (pp. 435-457). Museum Tusculanum Press.

Seyfarth, S., y Garellek, M. (2018). Plosive voicing acoustics and voice quality in Yerevan Armenian. Journal of Phonetics, 71, 425-450. https://doi.org/10.1016/j.wocn.2018.09.001

Smith-Stark, T. C. (2005). Phonological description in New Spain. En O. Zwartjes, C. Alt-man, y J. Calvo Pérez (Eds.), Missionary linguistics II. Lingüística misionera II. Or-thography and phonology (pp. 3-64). Ámsterdam: John Benjamins.

Sohn, H.-M. (2001). The Korean Language. Cambridge: Cambridge University Press.

Stark, L. R. (1985). The Quechua language in Bolivia. En H. E. Manelis Klein y L. R. Stark (Eds.), South American Indian languages (pp. 516-545). University of Texas Press.

Stark, L. R., y Muysken, P. C. (1977). Diccionario quichua-español y español-quichua. Banco Central del Ecuador.

Swadesh, M. (1952). Lexico-Statistic Dating of Prehistoric Ethnic Contacts: With Special Reference to North American Indians and Eskimos. Proceedings of the American Philosophical Society, 96(4), 452-463.

Taylor, G. (1979). Diccionario normalizado y comparativo quechua: Chachapoyas-Lamas. L'Harmattan.

Taylor, G. (2000). Estudios lingüísticos sobre Chachapoyas. Fondo Editorial de la Univer-sidad Nacional Mayor de San Marcos.

Taylor, G. (2001). Un sermón en quechua de Diego de Molina (Huánuco, 1649). Bulletin de I'Institut Français d'Études Andines, 30(2), 211-231. https://doi.org/10.4000/bifea.7032

Torero, A. (1964). Los dialectos quechuas. Anales Científicos de la Universidad Agraria, 2, 446-478.

Torero, A. (1972). Lingüística e historia de la sociedad andina. En A. Escobar (Ed.), El reto del multilingüismo en el Perú. Lima: Instituto de Estudios Peruanos.

Torero, A. (1984). El comercio lejano y la difusión del quechua El caso de Ecuador. Revista Andina, 2(2), 367-402.

Torero, A. (2002). Idiomas de los Andes: Lingüística e historia. Instituto Francés de Estu-dios Andinos.

Torres Rubio, D. de. (1616). Arte de la lengua aymara. Compuesta por el Padre Diego de Torres Rubio de la Compañia de lesus. Lima: Francisco del Canto. http://archive.org/details/ artedelalenguaay00torr

Torres Rubio, D. de. (1619). Arte de la lengua quichua. Lima: Francisco Lasso. http://archive. org/details/artedelalenguaqu04torr

Vail, H. L. (1972). Aspects of the Tumbuka Verb. University of Wisconsin.

Valqui, J. (2018). El acento en el quechua de Chachapoyas: Un sustrato de la lengua chacha o un vestigio protoquechua. Letras, 89(130), 79-99. https://doi.org/10.30920/letras.89.130.4

Valverde, J. A. (2015a). Derretroflexión y alveolarización en palabras quechuas I. Lengua y Sociedad, 15(1), 20-44.

Valverde, J. A. (2015b). Derretroflexión y alveolarización en palabras quechuas (II). Lengua y Sociedad, 15(2), 62-76.

Vergara y Vergara, J. M., y Delgado, E. (1860). The Indians of Andaqui, New Grenada. Bulletin American Ethnological Society, I, 53-72.

Yates, F. (1934). Contingency Tables Involving Small Numbers and the $\chi 2$ Test. Supple-ment to the Journal of the Royal Statistical Society, 1(2), 217-235. https://doi.org/10.2307/2983604 